\title{
Global analysis of three-flavour neutrino oscillations: synergies and tensions in the determination of $\theta_{23}$, $\delta_{\mathrm{CP}}$, and the mass ordering
}

\author{
Ivan Esteban, ${ }^{a}$ M.C. Gonzalez-Garcia, ${ }^{a, b, c}$ Alvaro Hernandez-Cabezudo, ${ }^{d}$ \\ Michele Maltoni $^{e}$ and Thomas Schwetz ${ }^{d}$ \\ ${ }^{a}$ Departament de Física Quàntica i Astrofísica and Institut de Ciencies del Cosmos, \\ Universitat de Barcelona, \\ Diagonal 647, E-08028 Barcelona, Spain \\ ${ }^{b}$ Institució Catalana de Recerca i Estudis Avançats (ICREA), \\ Pg. Lluis Companys 23, 08010 Barcelona, Spain \\ ${ }^{c}$ C.N. Yang Institute for Theoretical Physics, State University of New York at Stony Brook, \\ Stony Brook, NY 11794-3840, U.S.A. \\ ${ }^{d}$ Institut für Kernphysik, Karlsruher Institut für Technologie (KIT), \\ D-76021 Karlsruhe, Germany \\ ${ }^{e}$ Instituto de Física Teórica UAM/CSIC, Universidad Autónoma de Madrid, \\ Calle de Nicolás Cabrera 13-15, Cantoblanco, E-28049 Madrid, Spain \\ E-mail: ivan.esteban@fqa.ub.edu, \\ maria.gonzalez-garcia@stonybrook.edu, alvaro.cabezudo@kit.edu, \\ michele.maltoni@csic.es, schwetz@kit.edu
}

AbStRact: We present the results of a global analysis of the neutrino oscillation data available as of fall 2018 in the framework of three massive mixed neutrinos with the goal at determining the ranges of allowed values for the six relevant parameters. We describe the complementarity and quantify the tensions among the results of the different data samples contributing to the determination of each parameter. We also show how those vary when combining our global likelihood with the $\chi^{2}$ map provided by Super-Kamiokande for their atmospheric neutrino data analysis in the same framework. The best fit of the analysis is for the normal mass ordering with inverted ordering being disfavoured with a $\Delta \chi^{2}=4.7(9.3)$ without (with) SK-atm. We find a preference for the second octant of $\theta_{23}$, disfavouring the first octant with $\Delta \chi^{2}=4.4$ (6.0) without (with) SK-atm. The best fit for the complex phase is $\delta_{\mathrm{CP}}=215^{\circ}$ with $\mathrm{CP}$ conservation being allowed at $\Delta \chi^{2}=1.5(1.8)$. As a byproduct we quantify the correlated ranges for the laboratory observables sensitive to the absolute neutrino mass scale in beta decay, $m_{\nu_{e}}$, and neutrino-less double beta decay, $m_{e e}$, and the total mass of the neutrinos, $\Sigma$, which is most relevant in Cosmology.

Keywords: Neutrino Physics, Solar and Atmospheric Neutrinos

ARXIV EPRINT: 1811.05487 


\section{Contents}

1 Introduction $\quad 1$

2 Global analysis: determination of oscillation parameters 2

2.1 Data samples analyzed 2

2.2 Summary of global fit results 3

3 Synergies and tensions $\quad 8$

3.1 Status of comparison of results of solar experiments versus KamLAND 8

$3.2 \theta_{23}, \delta_{\mathrm{CP}}$ and mass ordering from LBL accelerator and MBL reactor experiments 10

$\begin{array}{lll}3.2 .1 & \text { Disappearance results and non-maximal } \theta_{23} & 10\end{array}$

3.2.2 Appearance results, second $\theta_{23}$ octant and $\delta_{\mathrm{CP}} \quad 13$

$\begin{array}{ll}3.2 .3 \text { Preference for normal ordering } & 15\end{array}$

3.3 Treatment of atmospheric results from Super-Kamiokande and Deep-Core 18

4 Projections on neutrino mass scale observables $\quad 20$

5 Conclusions $\quad 22$

A List of data used in the analysis $\quad 23$

B Technical details and validation cross checks $\quad 24$

$\begin{array}{lll}\text { B.1 T2K } & 25\end{array}$

B.2 NOvA 25

B.3 Daya Bay 26

$\begin{array}{lll}\text { B.4 RENO } 28 & 28\end{array}$

\section{Introduction}

Flavour transitions of neutrinos via the energy and distance dependent neutrino oscillation mechanism $[1,2]$ is a well established phenomenon, which proves that at least two out of the three neutrinos in the Standard Model must have tiny but non-zero masses. In this work we revisit the status of three-flavour neutrino oscillations in view of latest global data.

To fix the convention, the three flavour neutrinos, $\nu_{e}, \nu_{\mu}, \nu_{\tau}$, are defined via the weak charged current. They are expressed as superposition of the three neutrino mass eigen-fields $\nu_{i}(i=1,2,3)$ with masses $m_{i}$ via a unitary leptonic mixing matrix [3, 4] by

$$
\nu_{\alpha}=\sum_{i=1}^{3} U_{\alpha i} \nu_{i} \quad(\alpha=e, \mu, \tau) .
$$


The mixing matrix we parametrize as:

$$
U=\left(\begin{array}{ccc}
1 & 0 & 0 \\
0 & c_{23} & s_{23} \\
0 & -s_{23} & c_{23}
\end{array}\right) \cdot\left(\begin{array}{ccc}
c_{13} & 0 & s_{13} e^{-i \delta_{\mathrm{CP}}} \\
0 & 1 & 0 \\
-s_{13} e^{i \delta_{\mathrm{CP}}} & 0 & c_{13}
\end{array}\right) \cdot\left(\begin{array}{ccc}
c_{12} & s_{12} & 0 \\
-s_{12} & c_{12} & 0 \\
0 & 0 & 1
\end{array}\right) \cdot \mathcal{P}
$$

where $c_{i j} \equiv \cos \theta_{i j}$ and $s_{i j} \equiv \sin \theta_{i j}$. The angles $\theta_{i j}$ can be taken without loss of generality to lie in the first quadrant, $\theta_{i j} \in[0, \pi / 2]$, and the phase $\delta_{\mathrm{CP}} \in[0,2 \pi]$. Values of $\delta_{\mathrm{CP}}$ different from 0 and $\pi$ imply $\mathrm{CP}$ violation in neutrino oscillations in vacuum [5-7]. $\mathcal{P}$ is a diagonal matrix which is the identity if neutrinos are Dirac fermions and it contains two additional phases, $\mathcal{P}=\operatorname{diag}\left(e^{i \alpha_{1}}, e^{i \alpha_{2}}, 1\right)$, if they are Majorana fermions. The Majorana phases $\alpha_{1}$ and $\alpha_{2}$ play no role in neutrino oscillations [6, 8].

In this convention there are two non-equivalent orderings for the neutrino masses, namely normal ordering (NO) with $m_{1}<m_{2}<m_{3}$, and inverted ordering (IO) with $m_{3}<m_{1}<m_{2}$. Furthermore the data show a hierarchy between the mass splittings, $\Delta m_{21}^{2} \ll\left|\Delta m_{31}^{2}\right| \simeq\left|\Delta m_{32}^{2}\right|$ with $\Delta m_{i j}^{2} \equiv m_{i}^{2}-m_{j}^{2}$. In this work we follow the convention from ref. [9] and present our results for both, NO and IO, using the smallest and largest mass splittings. The smallest one is always $\Delta m_{21}^{2}$, while the largest one we denote by $\Delta m_{3 \ell}^{2}$, with $\ell=1$ for NO and $\ell=2$ for IO. Hence,

$$
\Delta m_{3 \ell}^{2}= \begin{cases}\Delta m_{31}^{2}>0 & \text { for } \mathrm{NO} \\ \Delta m_{32}^{2}<0 & \text { for IO }\end{cases}
$$

Due to the wealth of experiments exploring neutrino oscillations, we are in the situation that a given parameter is determined by several measurements. Therefore, combined analyses such as the one presented below are an important tool to extract the full information on neutrino oscillation parameters. This is especially true for open questions, such as the octant of $\theta_{23}$, the type of the neutrino mass ordering, and the status of the complex phase $\delta_{\mathrm{CP}}$, where some hints are emerging due to significant synergies between different experiments. However, also for parameters describing dominant oscillations, a significantly more accurate determination emerges by the combination of complementary data sets, such as for example for $\left|\Delta m_{3 \ell}^{2}\right|$.

We present below the global fit NuFIT-4.0, updating our previous analyses [9-11]. $\Delta \chi^{2}$ maps and future updates of this analysis will be made available at the NuFIT website [12]. For other recent global fits see $[13,14]$.

\section{Global analysis: determination of oscillation parameters}

\subsection{Data samples analyzed}

The analysis presented below uses data available up to fall 2018. A complete list of the used data including references can be found in appendix A. Here we give a brief overview of recent updates and mention changes with respect to our previous analysis [11].

We include latest data from the MINOS $[15,16]$, T2K $[17,18]$, and NOvA $[19,20]$ long-baseline accelerator experiments from $\nu_{\mu}$ disappearance and $\nu_{\mu} \rightarrow \nu_{e}$ appearance 
channels, both for neutrino and anti-neutrino beam modes. In particular, T2K and NOvA have presented updated results at the Neutrino18 conference, including also first data on anti-neutrinos from NOvA, whose impact will be discussed below.

Concerning reactor neutrino experiments, the fit of data with baselines in the $\mathrm{km}$ range (medium baseline, MBL) is completely dominated by modern experiments, most importantly by Daya Bay [21], with subleading contributions from RENO [22] and Double Chooz [23]. Moreover, those experiments are entirely based on relative spectra from detectors at different baselines, and are therefore largely independent of reactor neutrino flux predictions. In view of the unclear situation of reactor flux predictions and reactor data at very short baselines (see, e.g., ref. [24] for a recent discussion), we decided to include only the modern MBL reactor experiments Daya Bay, RENO, and Double Chooz. For the analysis of KamLAND long-baseline reactor data we replaced predicted neutrino fluxes by the spectrum measured in Daya Bay near detectors [25], which makes also our KamLAND analysis largely independent of flux predictions.

Our solar neutrino data includes previous data from radio-chemical and the SNO experiments, as well as updated exposures from Super-Kamiokande and Borexino, see appendix A for the detailed list and references. ${ }^{1}$

Atmospheric neutrino data generically are difficult to analyze outside the experimental collaborations. We present below two separate global analyses, depending on the used atmospheric neutrino data. Our default analysis makes use of IceCube/DeepCore 3-year data [27] which can be re-analyzed using the information provided by the collaboration [28]. Especially in the context of the mass ordering determination, atmospheric neutrino data from Super-Kamiokande 1-4 [29] seems to provide important information. Unfortunately there is not enough information available to reproduce these results outside the collaboration. However, Super-Kamiokande has published the results of their analysis in the form of a tabulated $\chi^{2}$ map [30], which we can combine with our global analysis. We will show the results of this combination as an alternative global fit. A detailed discussion of atmospheric neutrino data, including also the potential impact of an alternative IceCube analysis [31] will be presented in section 3.3.

\subsection{Summary of global fit results}

The results of our global fit are displayed in figure 1 (one-dimensional $\Delta \chi^{2}$ curves) and figure 2 (two-dimensional projections of confidence regions). In table 1 we give the best fit values as well as $1 \sigma$ and $3 \sigma$ confidence intervals for the oscillation parameters. We show two versions of the results. The default analysis is without Super-Kamiokande atmospheric neutrino data (SK-atm), and contains all the data for which a fit can be performed. For the alternative analysis, we add the pre-calculated $\Delta \chi^{2}$ table from SK-atm provided by the collaboration to our global fit, in order to illustrate the potential impact of these data. Let us summarize here the main features of the global fit result. More detailed discussions about how certain features emerge will be given in the following sections.

\footnotetext{
${ }^{1}$ We do not include here the latest data release from Borexino [26], which is expected to have a very small impact on the determination of oscillation parameters. These data will be included in future updates of our global fit.
} 

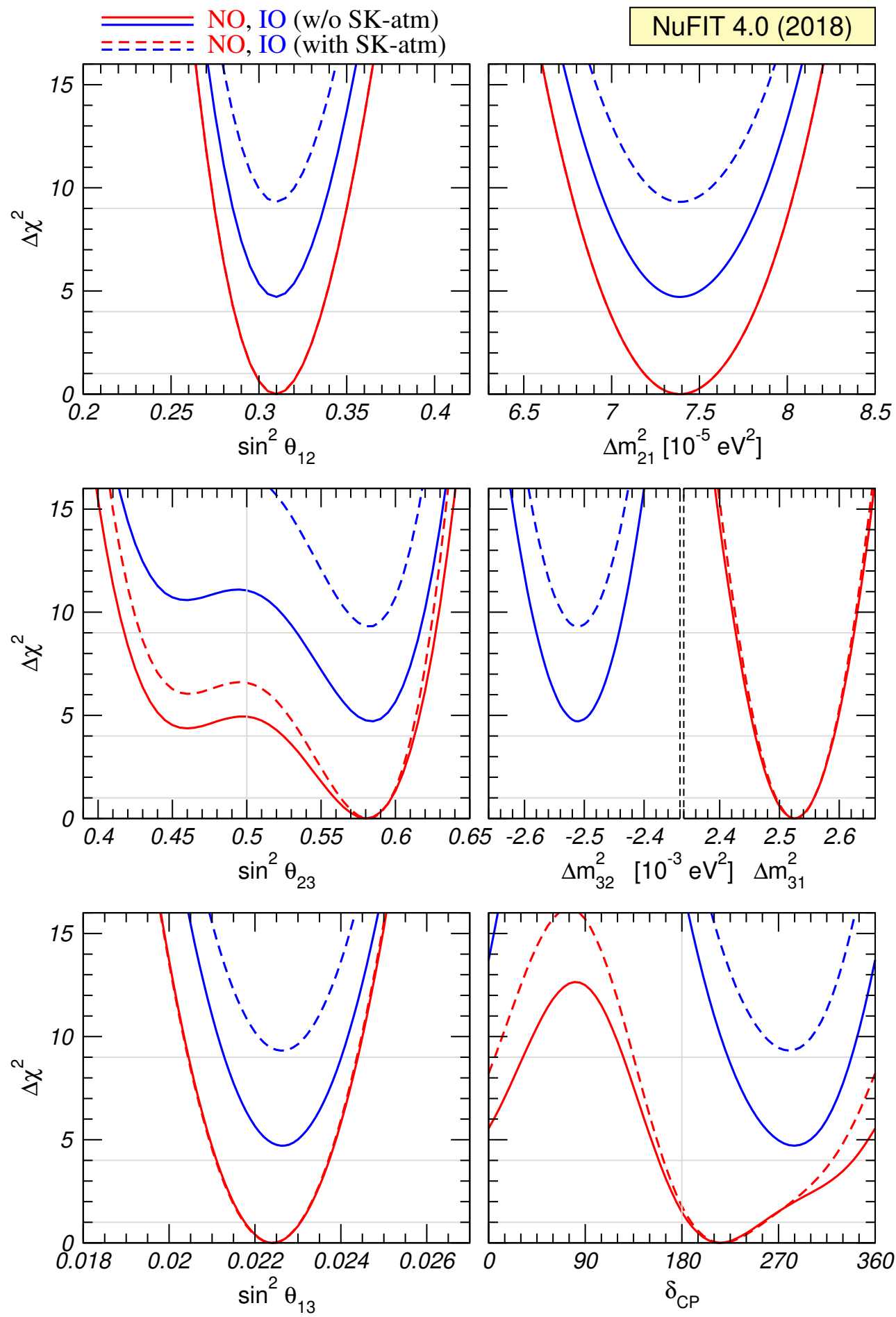

Figure 1. Global $3 \nu$ oscillation analysis. We show $\Delta \chi^{2}$ profiles minimized with respect to all undisplayed parameters. The red (blue) curves correspond to Normal (Inverted) Ordering. Solid (dashed) curves are without (with) adding the tabulated SK-atm $\Delta \chi^{2}$. Note that as atmospheric mass-squared splitting we use $\Delta m_{31}^{2}$ for NO and $\Delta m_{32}^{2}$ for IO. 


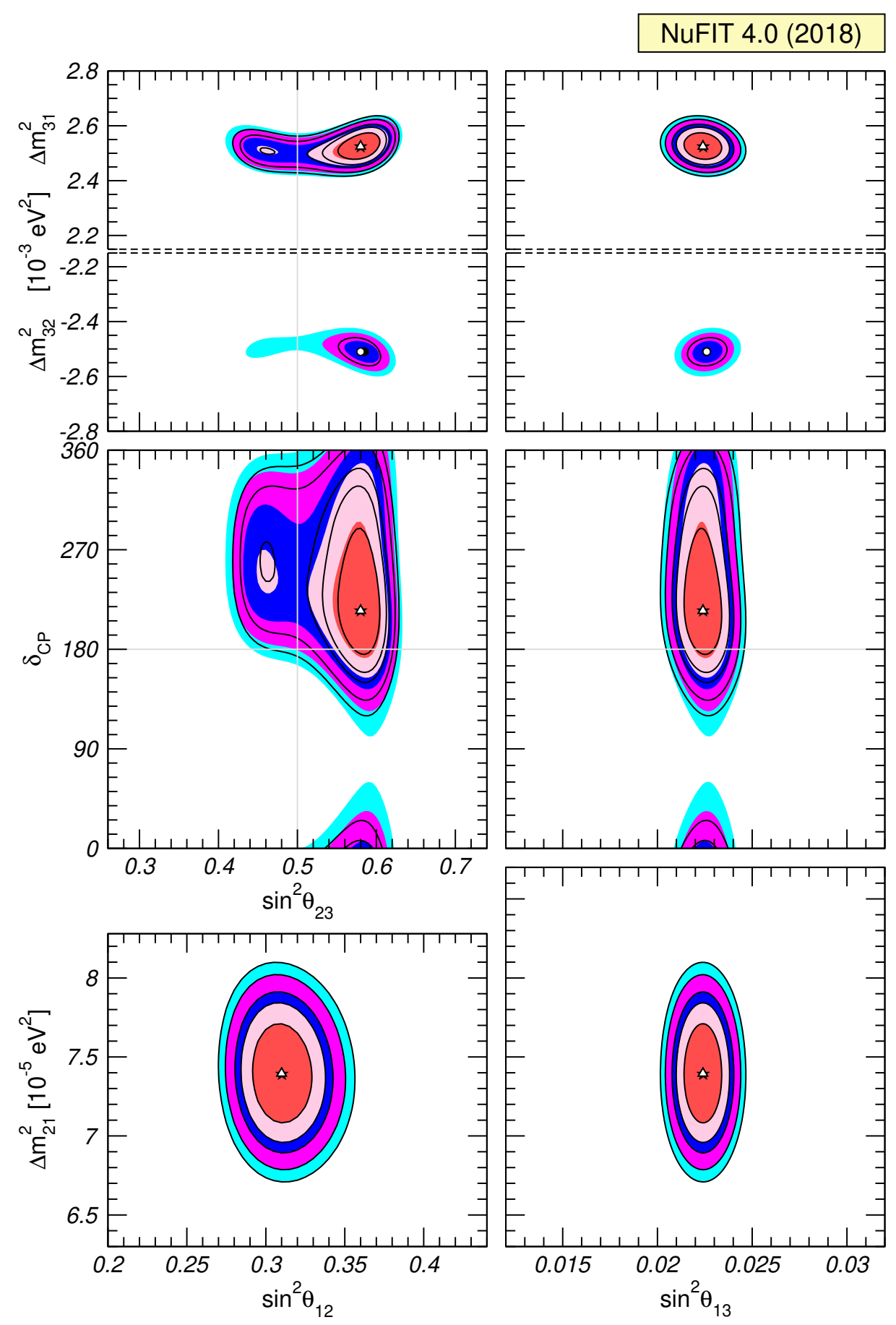

Figure 2. Global $3 \nu$ oscillation analysis. Each panel shows the two-dimensional projection of the allowed six-dimensional region after minimization with respect to the undisplayed parameters. The regions in the four lower panels are obtained from $\Delta \chi^{2}$ minimized with respect to the mass ordering. The different contours correspond to $1 \sigma, 90 \%, 2 \sigma, 99 \%, 3 \sigma$ CL ( 2 dof). Coloured regions (black contour curves) are without (with) adding the tabulated SK-atm $\Delta \chi^{2}$. Note that as atmospheric mass-squared splitting we use $\Delta m_{31}^{2}$ for NO and $\Delta m_{32}^{2}$ for IO. 


\begin{tabular}{|c|c|c|c|c|c|}
\hline \multirow{11}{*}{ 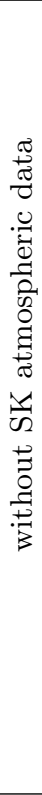 } & & \multicolumn{2}{|c|}{ Normal Ordering (best fit) } & \multicolumn{2}{|c|}{ Inverted Ordering $\left(\Delta \chi^{2}=4.7\right)$} \\
\hline & & $\mathrm{bfp} \pm 1 \sigma$ & $3 \sigma$ range & $\mathrm{bfp} \pm 1 \sigma$ & $3 \sigma$ range \\
\hline & $\sin ^{2} \theta_{12}$ & $0.310_{-0.012}^{+0.013}$ & $0.275 \rightarrow 0.350$ & $0.310_{-0.012}^{+0.013}$ & $0.275 \rightarrow 0.350$ \\
\hline & $\theta_{12} /^{\circ}$ & $33.82_{-0.76}^{+0.78}$ & $31.61 \rightarrow 36.27$ & $33.82_{-0.76}^{+0.78}$ & $31.61 \rightarrow 36.27$ \\
\hline & $\sin ^{2} \theta_{23}$ & $0.580_{-0.021}^{+0.017}$ & $0.418 \rightarrow 0.627$ & $0.584_{-0.020}^{+0.016}$ & $0.423 \rightarrow 0.629$ \\
\hline & $\theta_{23} /^{\circ}$ & $49.6_{-1.2}^{+1.0}$ & $40.3 \rightarrow 52.4$ & $49.8_{-1.1}^{+1.0}$ & $40.6 \rightarrow 52.5$ \\
\hline & $\sin ^{2} \theta_{13}$ & $0.02241_{-0.00065}^{+0.00065}$ & $0.02045 \rightarrow 0.02439$ & $0.02264_{-0.00066}^{+0.00066}$ & $0.02068 \rightarrow 0.02463$ \\
\hline & $\theta_{13} /^{\circ}$ & $8.61_{-0.13}^{+0.13}$ & $8.22 \rightarrow 8.99$ & $8.65_{-0.13}^{+0.13}$ & $8.27 \rightarrow 9.03$ \\
\hline & $\delta_{\mathrm{CP}} /^{\circ}$ & $215_{-29}^{+40}$ & $125 \rightarrow 392$ & $284_{-29}^{+27}$ & $196 \rightarrow 360$ \\
\hline & $\frac{\Delta m_{21}^{2}}{10^{-5} \mathrm{eV}^{2}}$ & $7.39_{-0.20}^{+0.21}$ & $6.79 \rightarrow 8.01$ & $7.39_{-0.20}^{+0.21}$ & $6.79 \rightarrow 8.01$ \\
\hline & $\frac{\Delta m_{3 \ell}^{2}}{10^{-3} \mathrm{eV}^{2}}$ & $+2.525_{-0.032}^{+0.033}$ & $+2.427 \rightarrow+2.625$ & $-2.512_{-0.032}^{+0.034}$ & $-2.611 \rightarrow-2.412$ \\
\hline \multirow{11}{*}{ 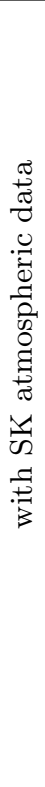 } & & \multicolumn{2}{|c|}{ Normal Ordering (best fit) } & \multicolumn{2}{|c|}{ Inverted Ordering $\left(\Delta \chi^{2}=9.3\right)$} \\
\hline & & $\mathrm{bfp} \pm 1 \sigma$ & $3 \sigma$ range & $\mathrm{bfp} \pm 1 \sigma$ & $3 \sigma$ range \\
\hline & $\sin ^{2} \theta_{12}$ & $0.310_{-0.012}^{+0.013}$ & $0.275 \rightarrow 0.350$ & $0.310_{-0.012}^{+0.013}$ & $0.275 \rightarrow 0.350$ \\
\hline & $\theta_{12} /^{\circ}$ & $33.82_{-0.76}^{+0.78}$ & $31.61 \rightarrow 36.27$ & $33.82_{-0.75}^{+0.78}$ & $31.62 \rightarrow 36.27$ \\
\hline & $\sin ^{2} \theta_{23}$ & $0.582_{-0.019}^{+0.015}$ & $0.428 \rightarrow 0.624$ & $0.582_{-0.018}^{+0.015}$ & $0.433 \rightarrow 0.623$ \\
\hline & $\theta_{23} /^{\circ}$ & $49.7_{-1.1}^{+0.9}$ & $40.9 \rightarrow 52.2$ & $49.7_{-1.0}^{+0.9}$ & $41.2 \rightarrow 52.1$ \\
\hline & $\sin ^{2} \theta_{13}$ & $0.02240_{-0.00066}^{+0.00065}$ & $0.02044 \rightarrow 0.02437$ & $0.02263_{-0.00066}^{+0.00065}$ & $0.02067 \rightarrow 0.02461$ \\
\hline & $\theta_{13} /^{\circ}$ & $8.61_{-0.13}^{+0.12}$ & $8.22 \rightarrow 8.98$ & $8.65_{-0.13}^{+0.12}$ & $8.27 \rightarrow 9.03$ \\
\hline & $\delta_{\mathrm{CP}} /^{\circ}$ & $217_{-28}^{+40}$ & $135 \rightarrow 366$ & $280_{-28}^{+25}$ & $196 \rightarrow 351$ \\
\hline & $\frac{\Delta m_{21}^{2}}{10^{-5} \mathrm{eV}^{2}}$ & $7.39_{-0.20}^{+0.21}$ & $6.79 \rightarrow 8.01$ & $7.39_{-0.20}^{+0.21}$ & $6.79 \rightarrow 8.01$ \\
\hline & $\frac{\Delta m_{3 \ell}^{2}}{10^{-3} \mathrm{eV}^{2}}$ & $+2.525_{-0.031}^{+0.033}$ & $+2.431 \rightarrow+2.622$ & $-2.512_{-0.031}^{+0.034}$ & $-2.606 \rightarrow-2.413$ \\
\hline
\end{tabular}

Table 1. Three-flavour oscillation parameters from our fit to global data. The numbers in the 1st (2nd) column are obtained assuming NO (IO), i.e., relative to the respective local minimum. Note that $\Delta m_{3 \ell}^{2} \equiv \Delta m_{31}^{2}>0$ for NO and $\Delta m_{3 \ell}^{2} \equiv \Delta m_{32}^{2}<0$ for IO. The results shown in the upper (lower) table are without (with) adding the tabulated SK-atm $\Delta \chi^{2}$.

Except for $\sin ^{2} \theta_{23}$ and $\delta_{\mathrm{CP}}$ the $\Delta \chi^{2}$ shapes are close to parabolic, indicating that the $\chi^{2}$ approximation for the distribution should hold to good accuracy. The Monte Carlo studies performed in refs. [11,32] indicate that also for $\sin ^{2} \theta_{23}, \delta_{\mathrm{CP}}$ and the mass ordering the $\chi^{2}$ approximation gives a reasonable estimate of the corresponding confidence level. Therefore, the $\Delta \chi^{2}$ values given below can be converted into an approximate number of standard deviations by the $\sqrt{\Delta \chi^{2}}$ rule. 
Defining the $3 \sigma$ relative precision of the parameter by $2\left(x^{\text {up }}-x^{\text {low }}\right) /\left(x^{\text {up }}+x^{\text {low }}\right)$, where $x^{\text {up }}\left(x^{\text {low }}\right)$ is the upper (lower) bound on a parameter $x$ at the $3 \sigma$ level, we obtain the following $3 \sigma$ relative precisions (marginalizing over ordering):

$$
\begin{array}{rlrlrl}
\theta_{12} & : 14 \%, & \theta_{13} & : 8.9 \%, & \theta_{23}: 27 \%[24 \%], \\
\Delta m_{21}^{2}: 16 \%, & \left|\Delta m_{3 \ell}^{2}\right|: 7.8 \%[7.6 \%], & \delta_{\mathrm{CP}}: 100 \%[92 \%],
\end{array}
$$

where the numbers between brackets show the impact of including SK-atm in the precision of that parameter determination. We notice that as $\Delta \chi^{2}$ shape for $\delta_{\mathrm{CP}}$ is clearly not gaussian this evaluation of its "precision" can only be taken as indicative.

Altogether the status of mass ordering discrimination, determination of $\sin ^{2} \theta_{23}$, and the leptonic $\mathrm{CP}$ phase $\delta_{\mathrm{CP}}$ can be summarized as follows:

- The best fit is for the normal mass ordering. Inverted ordering is disfavoured with a $\Delta \chi^{2}=4.7(9.3)$ without (with) SKatm.

- We obtain preference for the second octant of $\theta_{23}$, with the best fit point located at $\sin ^{2} \theta_{23}=0.58$. Values with $\sin ^{2} \theta_{23} \leq 0.5$ are disfavoured with $\Delta \chi^{2}=4.4(6.0)$ without (with) SK-atm.

- The best fit for the complex phase is at $\delta_{\mathrm{CP}}=215^{\circ}$. Compared to previous results (e.g., NuFIT $3.2[12]$ ), the allowed range is pushed towards the CP conserving value of $180^{\circ}$, which now is only disfavoured with $\Delta \chi^{2}=1.5$ (1.8) without (with) SK-atm.

In table 1 we give the best fit values and confidence intervals for both mass orderings, relative to the local best fit points in each ordering. The global confidence intervals (marginalizing also over the ordering) are identical to the ones for normal ordering, which have also been used in eq. (2.1). The only exception to this statement is $\Delta m_{3 \ell}^{2}$ in the analysis without SK-atm; in this case a disconnected interval would appear above $2 \sigma$ corresponding to negative values of $\Delta m_{3 \ell}^{2}$ (i.e., inverted ordering).

Let us briefly compare our results to those from other groups [13, 14], noting however, that the data samples used in those references are not the same as in our analysis. Our results are in good agreement with those in refs. $[13,14]$, in particular for the allowed ranges of the most precisely determined parameters, $\Delta m_{21}^{2}, \theta_{12}, \theta_{13}$ and $\Delta m_{3 \ell}^{2}$. Also all groups find $\mathcal{O}(3 \sigma)$ preference for NO when including SK-atm results and slight favouring of the second octant of $\theta_{23}$. The main quantitative variation of our present results in comparison with those in $[13,14]$ concerns the allowed range of $\delta_{\mathrm{CP}}$ and the confidence level for CP conservation and for maximal $\theta_{23}$, and it is mostly driven by the inclusion of the latest NOvA results in our analysis (see discussion in the next section).

Altogether we derive the following $3 \sigma$ ranges on the magnitude of the elements of the leptonic mixing matrix:

$$
\begin{aligned}
|U|_{3 \sigma}^{\text {w/o SK-atm }} & =\left(\begin{array}{lll}
0.797 \rightarrow 0.842 & 0.518 \rightarrow 0.585 & 0.143 \rightarrow 0.156 \\
0.233 \rightarrow 0.495 & 0.448 \rightarrow 0.679 & 0.639 \rightarrow 0.783 \\
0.287 \rightarrow 0.532 & 0.486 \rightarrow 0.706 & 0.604 \rightarrow 0.754
\end{array}\right) \\
|U|_{3 \sigma}^{\text {with SK-atm }} & =\left(\begin{array}{lll}
0.797 \rightarrow 0.842 & 0.518 \rightarrow 0.585 & 0.143 \rightarrow 0.156 \\
0.235 \rightarrow 0.484 & 0.458 \rightarrow 0.671 & 0.647 \rightarrow 0.781 \\
0.304 \rightarrow 0.531 & 0.497 \rightarrow 0.699 & 0.607 \rightarrow 0.747
\end{array}\right)
\end{aligned}
$$



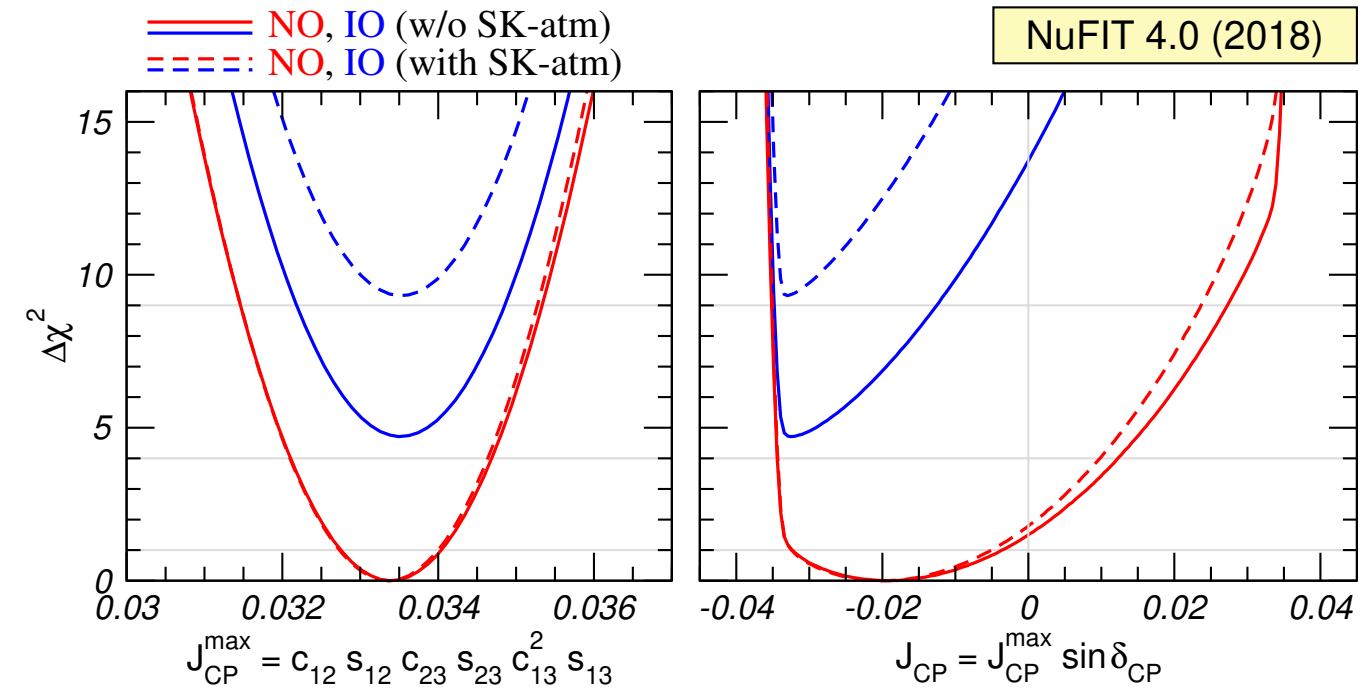

Figure 3. Dependence of the global $\Delta \chi^{2}$ function on the Jarlskog invariant. The red (blue) curves are for NO (IO). Solid (dashed) curves are without (with) adding the tabulated SK-atm $\Delta \chi^{2}$.

Note that there are strong correlations between the elements due to the unitary constraint, see ref. [33] for details on how we derive the ranges.

The present status of leptonic CP violation is illustrated in figures 2 and 3. In particular, figure 2 contains two projections of the confidence regions with $\delta_{\mathrm{CP}}$ on the vertical axis in which we observe the non-trivial correlations between $\delta_{\mathrm{CP}}$ and $\sin ^{2} \theta_{23}$. In the left panel of figure 3 we show the dependence of $\Delta \chi^{2}$ of the global analysis on the Jarlskog invariant which gives a convention-independent measure of leptonic $\mathrm{CP}$ violation in neutrino propagation in vacuum [34] - analogous to the factor introduced in ref. [35] for the description of $\mathrm{CP}$ violating effects in the quark sector — defined by:

$$
\begin{aligned}
J_{\mathrm{CP}} & \equiv \operatorname{Im}\left[U_{\alpha i} U_{\alpha j}^{*} U_{\beta i}^{*} U_{\beta j}\right] \\
& \equiv J_{\mathrm{CP}}^{\max } \sin \delta_{\mathrm{CP}}=\cos \theta_{12} \sin \theta_{12} \cos \theta_{23} \sin \theta_{23} \cos ^{2} \theta_{13} \sin \theta_{13} \sin \delta_{\mathrm{CP}}
\end{aligned}
$$

where in the second line we have used the parametrization in eq. (1.2). Factoring out $\sin \delta_{\mathrm{CP}}$, the determination of the mixing angles implies a maximal possible value of the Jarlskog invariant:

$$
J_{\mathrm{CP}}^{\max }=0.0333 \pm 0.0006( \pm 0.0019)
$$

at $1 \sigma(3 \sigma)$ for both orderings. The preference of the present data for non-zero $\delta_{\mathrm{CP}}$ implies a best fit value $J_{\mathrm{CP}}^{\text {best }}=-0.019$, which is favored over CP conservation with $\Delta \chi^{2}=1.5(1.8)$ without (with) SK-atm. These numbers can be compared with the size of the Jarlskog invariant in the quark sector, $J_{\mathrm{CP}}^{\text {quarks }}=(3.18 \pm 0.15) \times 10^{-5}[36]$.

\section{Synergies and tensions}

\subsection{Status of comparison of results of solar experiments versus KamLAND}

The analyses of the solar experiments and of KamLAND give the dominant contribution to the determination of $\Delta m_{21}^{2}$ and $\theta_{12}$. We show in figure 4 the present determination of these 
parameters from the global solar analysis in comparison with that of KamLAND data. The results of the solar neutrino analysis are shown for the two latest versions of the Standard Solar Model, namely the GS98 and the AGSS09 models [37] obtained with two different determinations of the solar abundances [38]. This clearly illustrates the independence of the results with respect to the solar modeling.

There are two main differences compared to our previous published results in ref. [11]. In what respects the KamLAND region it has shifted towards slightly smaller values of $\theta_{12}$. This effect arises mainly from the new reactor fluxes used in our analysis of the KamLAND data. As mentioned in section 2.1, in our calculation of the event rates in KamLAND we have replaced the predicted neutrino fluxes by the spectrum measured in Daya Bay near detectors [25] which is unfolded for detector and remaining oscillation effects. In ref. [11] we used instead the unoscillated reactor determined by including in the fit the results from a compilation of short baseline reactor data. The net result is that the current unoscillated reactor fluxes are slightly lower and consequently a slightly higher survival probability is required to better fit the data. Since in the context of $3 \nu$-oscillations

$$
P_{e e, \mathrm{KLAND}}^{3 \nu}=\sin ^{4} \theta_{13}+\cos ^{4} \theta_{13}\left(1-\frac{1}{2} \sin ^{2}\left(2 \theta_{12}\right) \sin ^{2} \frac{\Delta m_{21}^{2} L}{2 E}\right)
$$

a larger survival probability implies smaller values of $\theta_{12}$. As a result the best-fit value of $\theta_{12}$ determined by KamLAND, $\sin ^{2} \theta_{12 \text {,bf-Kam }}=0.290$, does not perfectly align with the corresponding best fit value from the solar neutrino analysis, $\sin ^{2} \theta_{12 \text {,bf-sol }}=0.315$. Statistically, however, this is a very small effect as the best fit value of $\sin ^{2} \theta_{12}=0.315$ lies at $\Delta \chi_{\text {KamLAND }}^{2} \lesssim 1$

In what respects the determination of $\Delta m_{21}^{2}$ it has been a result of global analyses for several years already, that the value of $\Delta m_{21}^{2}$ preferred by KamLAND is somewhat higher than the one from solar experiments. The tension arises from a combination of two effects: the well-known fact that none of the ${ }^{8} \mathrm{~B}$ measurements performed by SNO, SK and Borexino shows any evidence of the low energy spectrum turn-up expected in the standard LMA-MSW [39, 40] solution for the value of $\Delta m_{21}^{2}$ favored by KamLAND; and the observation of a non-vanishing day-night asymmetry in SK, whose size is larger than the one predicted for the $\Delta m_{21}^{2}$ value indicated of KamLAND.

The new addition to this issue in the present analysis is the inclusion of the 2860-day energy spectrum of SK4 [41] (compared to the 2365-day energy spectrum used in [11]). For the day-night variation of the results we still use the SK4 2055-day day-night asymmetry [42] because SK has not presented any update concerning the day-night dependence of the observed rates. The inclusion of the new spectral data makes the lack of the turn-up effect slightly stronger (for example the best fit $\Delta m_{21}^{2}$ of KamLAND was at $\Delta \chi_{\text {solar }}^{2}=4$ in the analysis of [11] with the GS98 fluxes and it is now at $\left.\Delta \chi_{\text {solar }}^{2}=4.7\right)$. For illustration of the relevance of the day-night variation results we plot in figure 4 the corresponding results of the solar analysis without including the day-night asymmetry. 

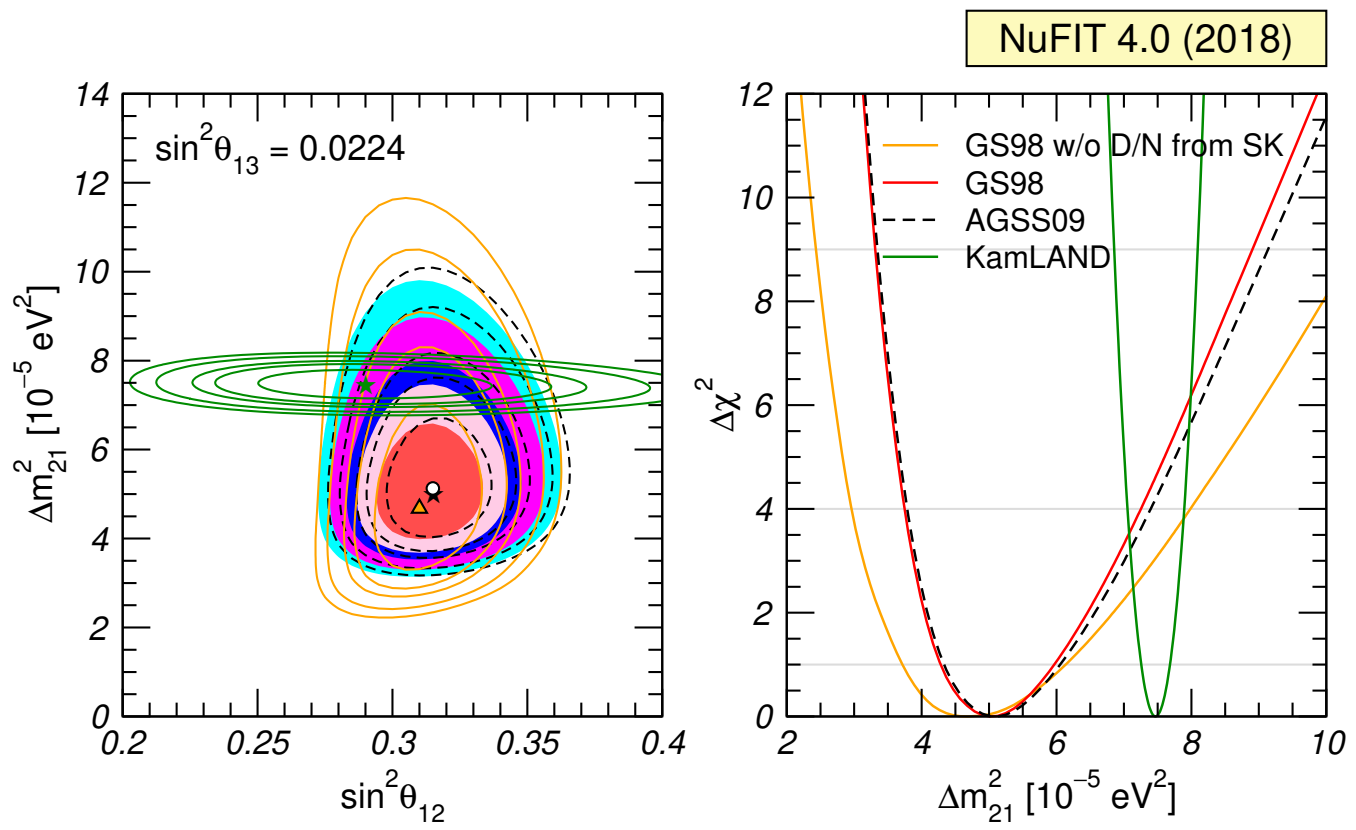

Figure 4. Left: allowed parameter regions (at $1 \sigma, 90 \%, 2 \sigma, 99 \%$, and $3 \sigma$ CL for 2 dof) from the combined analysis of solar data for GS98 model (full regions with best fit marked by black star) and AGSS09 model (dashed void contours with best fit marked by a white dot), and for the analysis of KamLAND data (solid green contours with best fit marked by a green star) for fixed $\sin ^{2} \theta_{13}=0.0224\left(\theta_{13}=8.6\right)$. We also show as orange contours the results of a global analysis for the GS98 model but without including the day-night information from SK. Right: $\Delta \chi^{2}$ dependence on $\Delta m_{21}^{2}$ for the same four analyses after marginalizing over $\theta_{12}$.

\section{$3.2 \theta_{23}, \delta_{\mathrm{CP}}$ and mass ordering from LBL accelerator and MBL reactor experiments}

The determination of the atmospheric parameters $\theta_{23}$ and $\Delta m_{3 \ell}^{2}$ is illustrated in figure 5 . We observe significant synergy from combining the various experiments, since the combined region is clearly smaller than any individual one. Moreover, the striking agreement of LBL accelerator and MBL reactor data in the determination of $\Delta m_{3 \ell}^{2}$ within comparable accuracy is a non-trivial cross check of the 3-flavour oscillation paradigm. Let us now discuss in more detail how the indication of non-maximal mixing and preference for the second octant for $\theta_{23}$ emerges.

\subsubsection{Disappearance results and non-maximal $\theta_{23}$}

We focus first on LBL disappearance data. The $\nu_{\mu}$ survival probability is given to good accuracy by $[43,44]$

$$
P_{\mu \mu} \approx 1-\sin ^{2} 2 \theta_{\mu \mu} \sin ^{2} \frac{\Delta m_{\mu \mu}^{2} L}{4 E_{\nu}},
$$

where $L$ is the baseline, $E_{\nu}$ is the neutrino energy, and

$$
\begin{aligned}
\sin ^{2} \theta_{\mu \mu} & =\cos ^{2} \theta_{13} \sin ^{2} \theta_{23}, \\
\Delta m_{\mu \mu}^{2} & =\sin ^{2} \theta_{12} \Delta m_{31}^{2}+\cos ^{2} \theta_{12} \Delta m_{32}^{2}+\cos \delta_{\mathrm{CP}} \sin \theta_{13} \sin 2 \theta_{12} \tan \theta_{23} \Delta m_{21}^{2} .
\end{aligned}
$$




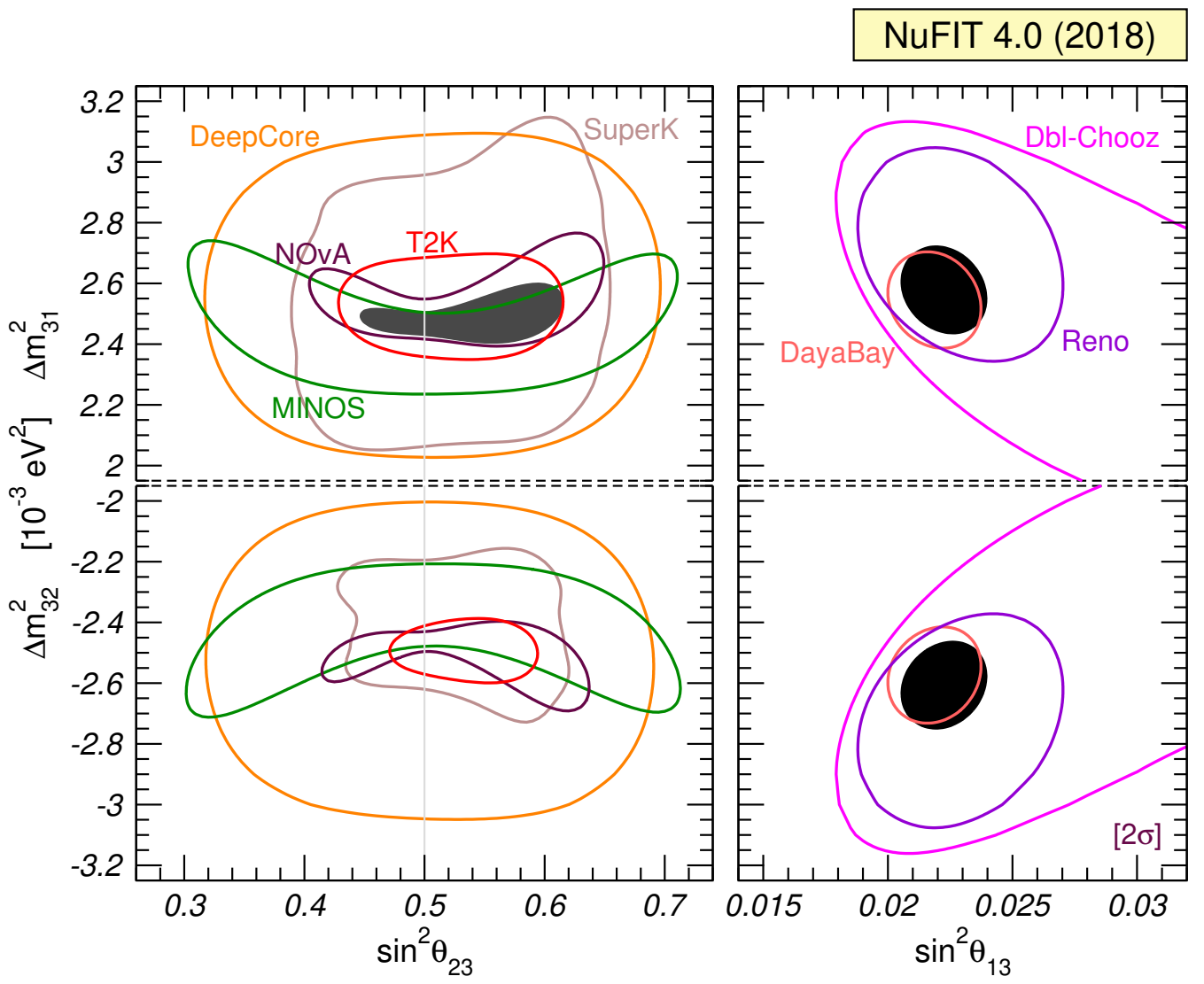

Figure 5. Determination of $\Delta m_{3 \ell}^{2}$ at $2 \sigma$ (2 dof), where $\ell=1$ for NO (upper panels) and $\ell=2$ for IO (lower panels). The left panels show regions in the $\left(\theta_{23}, \Delta m_{3 \ell}^{2}\right)$ plane using both appearance and disappearance data from MINOS (green), T2K (red), NOvA (dark-redwood), as well as IceCube/DeepCore (orange), and SK-atm (from the table provided by the experiment, light-brown line) and the combination of them (dark-grey coloured region). In the left panels the constraint on $\theta_{13}$ from the global fit (which is dominated by the reactor data) is imposed as a Gaussian bias. The right panels show regions in the $\left(\theta_{13}, \Delta m_{3 \ell}^{2}\right)$ plane using only Daya Bay (pink), Reno (violet) and Double Chooz (magenta) reactor data, and their combination (black coloured region). In all panels $\Delta m_{21}^{2}, \sin ^{2} \theta_{12}$ are fixed to the global best fit values. Contours are defined with respect to the global minimum of the two orderings.

Hence the survival probability is symmetric with respect to the octant of $\theta_{\mu \mu}$, which implies symmetry around $s_{23}^{2}=0.5 / c_{13}^{2} \approx 0.51$. This behaviour is visible in the left panels of figure 6, which show the results of LBL accelerator disappearance data from MINOS, T2K, NOvA, separated into the neutrino and anti-neutrino data samples (for fixed value of $\theta_{13}$ at the best fit and NO). While most of the shown data samples prefer maximal mixing (especially T2K and NOvA neutrino data), maximal mixing is disfavoured by MINOS neutrino data $\left(\Delta \chi^{2} \approx 2\right)$ and NOvA anti-neutrino data $\left(\Delta \chi^{2} \approx 6\right)$. This behaviour can be traced back to the number of events in the corresponding data samples observed at the dip of the survival probability: for maximal mixing the survival probability is zero at the dip and no events should be observed. Qualitatively similar behaviour are found for IO. 

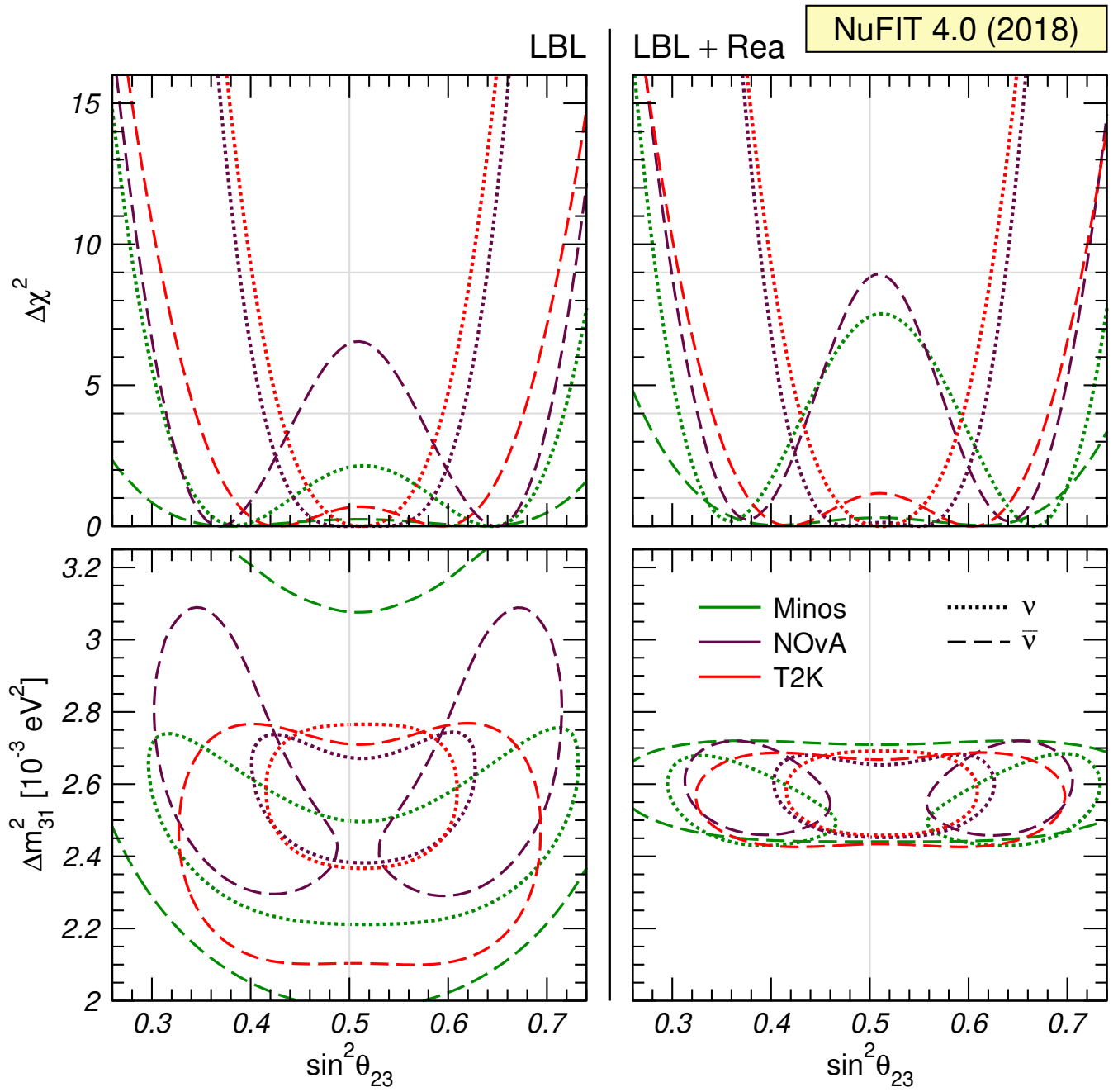

Figure 6. LBL accelerator $\nu_{\mu}$ disappearance data only, from MINOS, T2K, and NOvA, separated into neutrino and anti-neutrino data. Left panels correspond to LBL accelerator data with constraint on $\theta_{13}$ from the global fit (which is dominated by the MBL reactor data) imposed as a Gaussian bias. In the right panels LBL data are consistently combined with MBL reactor data from Daya Bay, RENO, and Double Chooz. Upper panels show the $\Delta \chi^{2}$ as a function of $\sin ^{2} \theta_{23}$, lower panels show confidence regions at $2 \sigma$ (2 dof). All panels assume NO and $\Delta m_{21}^{2}, \sin ^{2} \theta_{12}$ are fixed to the global best fit values. Qualitatively similar behaviour is found in IO.

In the lower-left panel of figure 6 we observe in addition a correlation between $\sin ^{2} \theta_{23}$ and $\Delta m_{31}^{2}$ for the data which prefer non-maximal mixing: larger values of $\Delta m_{31}^{2}$ imply more deviation from maximal mixing. As visible in figure 5, also MBL reactor data provide an accurate determination of $\Delta m_{3 \ell}^{2}$, which, however, pushes slightly to larger values than LBL data. Because of the above mentioned correlation, this leads to an even stronger preference for non-maximal mixing, once LBL data are consistently combined with reactor data, as visible in the right panels of figure 6 : in combination with reactors, MINOS neutrino and NOvA anti-neutrino data disfavour maximal mixing with $\Delta \chi^{2} \approx 7$ and 9 , respectively. 


\subsubsection{Appearance results, second $\theta_{23}$ octant and $\delta_{\mathrm{CP}}$}

The preference for the second octant of $\theta_{23}$ is driven by $\nu_{\mu} \rightarrow \nu_{e}$ appearance channel in LBL experiments (available both for neutrinos and anti-neutrinos). Following ref. [32], the appearance probability can be approximated by

$$
\begin{aligned}
& P_{\nu_{\mu} \rightarrow \nu_{e}} \approx 4 s_{13}^{2} s_{23}^{2}(1+2 o A)-C \sin \delta_{\mathrm{CP}}(1+o A), \\
& P_{\bar{\nu}_{\mu} \rightarrow \bar{\nu}_{e}} \approx 4 s_{13}^{2} s_{23}^{2}(1-2 o A)+C \sin \delta_{\mathrm{CP}}(1-o A),
\end{aligned}
$$

with

$$
C \equiv \frac{\Delta m_{21}^{2} L}{4 E_{\nu}} \sin 2 \theta_{12} \sin 2 \theta_{13} \sin 2 \theta_{23}, \quad o \equiv \operatorname{sgn}\left(\Delta m_{3 \ell}^{2}\right), \quad A \equiv\left|\frac{2 E_{\nu} V}{\Delta m_{3 \ell}^{2}}\right|,
$$

where $V$ is the effective matter potential. In the above equations we have expanded in the small parameters $s_{13}, \Delta m_{21}^{2} L / E_{\nu}$, and $A$, and used that for T2K and NOvA $\left|\Delta m_{3 \ell}^{2}\right| L / 4 E_{\nu} \approx \pi / 2 .^{2}$ Using the respective mean neutrino energies we find $A \approx 0.05$ for $\mathrm{T} 2 \mathrm{~K}$ and an empirical value of $A=0.1$ (for which this approximation works better) at NOvA. Correspondingly the number of observed appearance events in T2K and NOvA is approximately proportional to the oscillation probability:

$$
\begin{aligned}
& N_{\nu_{e}} \approx \mathcal{N}_{\nu}\left[2 s_{23}^{2}(1+2 o A)-C^{\prime} \sin \delta_{\mathrm{CP}}(1+o A)\right], \\
& N_{\bar{\nu}_{e}} \approx \mathcal{N}_{\bar{\nu}}\left[2 s_{23}^{2}(1-2 o A)+C^{\prime} \sin \delta_{\mathrm{CP}}(1-o A)\right] .
\end{aligned}
$$

Taking all the well-determined parameters $\theta_{13}, \theta_{12}, \Delta m_{21}^{2},\left|\Delta m_{3 \ell}^{2}\right|$ at their global best fit points, we obtain numerically $C^{\prime} \approx 0.28$. The normalization constants $\mathcal{N}_{\nu, \bar{\nu}}$ calculated from our re-analysis of $\mathrm{T} 2 \mathrm{~K}$ and NOvA are given for the various appearance samples in table 2. Those values can be compared with the background subtracted observed number of events, which we also report in the table. Within this approximation, there are only the two parameters $s_{23}^{2}$ and $\sin \delta_{\mathrm{CP}}$, plus the discrete parameter $o= \pm 1$ encoding the mass ordering, to fit the appearance event numbers shown in table 2 , with $\sin ^{2} \theta_{23}$ being constrained in addition from disappearance data. Note that $C^{\prime}$ depends only on $\sin 2 \theta_{23}$, which varies by less than $2 \%$ for $0.42<s_{23}^{2}<0.64$, and can be taken as constant for our purposes. The general trends from eqs. (3.8) and (3.9) are the following:

- Both neutrino and anti-neutrino events are enhanced by increasing $s_{23}^{2}$.

- Values of $\sin \delta_{\mathrm{CP}} \simeq+1(-1)$ suppress (increase) neutrino events, and have the opposite effect for anti-neutrino events.

- For NO (IO) neutrino events are enhanced (suppressed) due to the matter effect, whereas anti-neutrino events are suppressed (enhanced).

- For NO (IO) the matter effect increases (decreases) the impact of $\delta_{\mathrm{CP}}$ for neutrinos, while the opposite happens for anti-neutrinos.

\footnotetext{
${ }^{2}$ Expanding in the matter potential parameter $A$ is a very good approximation for T2K, but not so good for NOvA. However, the qualitative behaviour is still captured by the above expressions also for NOvA, which suffices for our discussion here.
} 


\begin{tabular}{|c|ccccc|}
\hline & T2K CCQE $(\nu)$ & T2K CC1 $\pi(\nu)$ & T2K CCQE $(\bar{\nu})$ & NOvA $(\nu)$ & NOvA $(\bar{\nu})$ \\
\hline $\mathcal{N}$ & 40 & 3.8 & 11 & 34 & 11 \\
$N_{\text {obs }}$ & 75 & 15 & 9 & 58 & 18 \\
$N_{\text {obs }}-N_{\text {bck }}$ & 61.4 & 13.6 & 6.1 & 43.6 & 13.8 \\
\hline
\end{tabular}

Table 2. Normalization coefficients $\mathcal{N}_{\nu}$ and $\mathcal{N}_{\bar{\nu}}$ for eqs. (3.8) and (3.9) for approximations used to qualitatively describe the various appearance event samples used in our analysis for $\mathrm{T} 2 \mathrm{~K}$ and NOvA. We also give the observed number of events, as well as the corresponding background subtracted event numbers, as reported in refs. [45, 46].

The last two items are more important for NOvA than for T2K, due to larger matter effects in NOvA because of the longer baseline.

In figure 7 , the determination of $s_{23}^{2}$ from LBL data (including appearance) combined with reactor data is shown. In the upper panels only $\theta_{13}$ is constrained by reactor data, whereas in the lower panels LBL and reactor data are combined consistently, including also $\Delta m_{3 \ell}^{2}$ information. For the reasons explained above, lower panels show larger significance of non-maximality, but now the symmetry between the octants is broken by appearance data. Figure 8 shows the $\Delta \chi^{2}$ dependence on $\delta_{\mathrm{CP}}$ for various data samples.

Let us consider first the $\mathrm{T} 2 \mathrm{~K}$ samples. We see from table 2 that in both neutrino samples (especially $\mathrm{CC} 1 \pi$ ) the observed number of events after background subtraction is large compared to $\mathcal{N}_{\nu}$, while the anti-neutrino number is low. Hence, we need to maximize the expression in eq. (3.8) and minimize eq. (3.9). Since neutrino data dominates over antineutrinos, a slight preference for $s_{23}^{2}>0.5$ appears (constrained by disappearance data), while at the same time $\sin \delta_{\mathrm{CP}} \approx-1$ serves to maximize (minimize) neutrino (anti-neutrino) appearance, as visible in figure 8 .

For NOvA neutrino data, the coefficient $\mathcal{N}_{\nu}$ in eq. (3.8) is also somewhat low compared to the observed number of events minus background. For NO, the matter effect enhances neutrino events, and therefore, $s_{23}^{2}$ (around maximal mixing favoured in disappearance) and $\delta_{\mathrm{CP}}$ can be adjusted, such that the event numbers can always be fitted, so $\Delta \chi^{2}\left(\delta_{\mathrm{CP}}\right)$ from NOvA neutrino data alone is $<1$ for NO, cf. figure 8. For IO, however, the matter effect suppresses neutrino events, and therefore, preference for the second octant and $\sin \delta_{\mathrm{CP}} \approx-1$ appears to maximize the term in the square-bracket in eq. (3.8). For NOvA anti-neutrino data, table 2 shows that the observed event number is of the order of $\mathcal{N}_{\bar{\nu}}$ (only slightly higher). Consequently we observe for NO only a very mild preference for $\sin \delta_{\mathrm{CP}} \approx 1$ just to enhance slightly the rate of anti-neutrinos. For IO, the matter effect enhances anti-neutrinos, and therefore, choosing the combinations (first $\theta_{23}$ octant $/ \sin \delta_{\mathrm{CP}} \approx 1$ ) or (second $\theta_{23}$ octant $/ \sin \delta_{\mathrm{CP}} \approx-1$ ) can fit the events, which leads to negligible $\Delta \chi^{2}\left(\delta_{\mathrm{CP}}\right)$ dependence for IO NOvA anti-neutrinos, cf. figure 8 . The combination of those effects for $\mathrm{NO}$, leads to a disfavouring of $\sin \delta_{\mathrm{CP}} \approx-1$ with $\Delta \chi^{2} \approx 3.5$ from NOvA, somewhat in contradiction of the $\mathrm{T} 2 \mathrm{~K}$ preferred region: with the non-maximality of $\theta_{23}$ from anti-neutrinos plus the matter enhancement for neutrinos, $\sin \delta_{\mathrm{CP}} \approx-1$ would predict too many neutrino events, and is therefore disfavoured. 

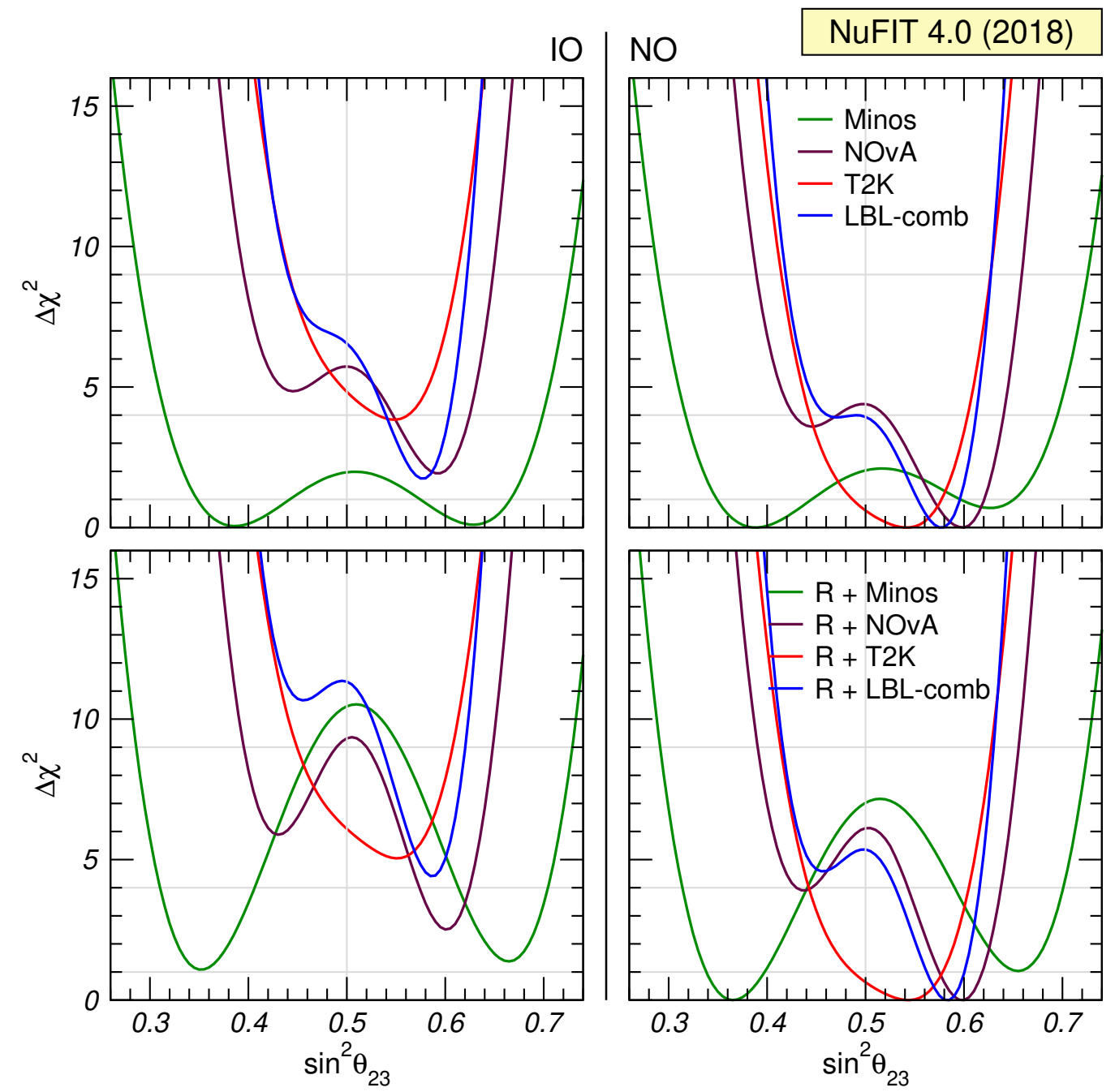

Figure 7. $\theta_{23}$ determination from LBL, reactor and their combination. Left (right) panels are for IO (NO). The upper panels show the 1-dim $\Delta \chi^{2}$ from LBL experiments after constraining only $\theta_{13}$ from reactor experiments. For each experiment $\Delta \chi^{2}$ is defined with respect to the global minimum of the two orderings. The lower panels show the corresponding determination when the full information of LBL accelerator and reactor experiments is used in the combination (including the information on $\Delta m_{3 \ell}^{2}$ from reactors). In all panels $\Delta m_{21}^{2}, \sin ^{2} \theta_{12}$ are fixed to the global best fit values.

The conclusion of those considerations lead to the preference of the second octant for $\theta_{23}$ in the global analysis, as well as pushing the confidence interval for $\delta_{\mathrm{CP}}$ towards $180^{\circ}$, which implies that $\mathrm{CP}$ conservation is allowed by the combined data with $\Delta \chi^{2} \approx 1.5$.

\subsubsection{Preference for normal ordering}

An important result of the present global fit is the growing significance of the preference for the normal mass ordering. This indication emerges by a subtle interplay of various subsets of the global data. Sensitivity to the mass ordering is provided by the matter effect [39, $40,47]$ in oscillations with $\Delta m_{3 \ell}^{2}$, observable in LBL accelerator and atmospheric neutrino experiments, as well as the comparison of oscillations in the $\nu_{e}$ and $\nu_{\mu}$ disappearance channels [44, 48, 49]. 

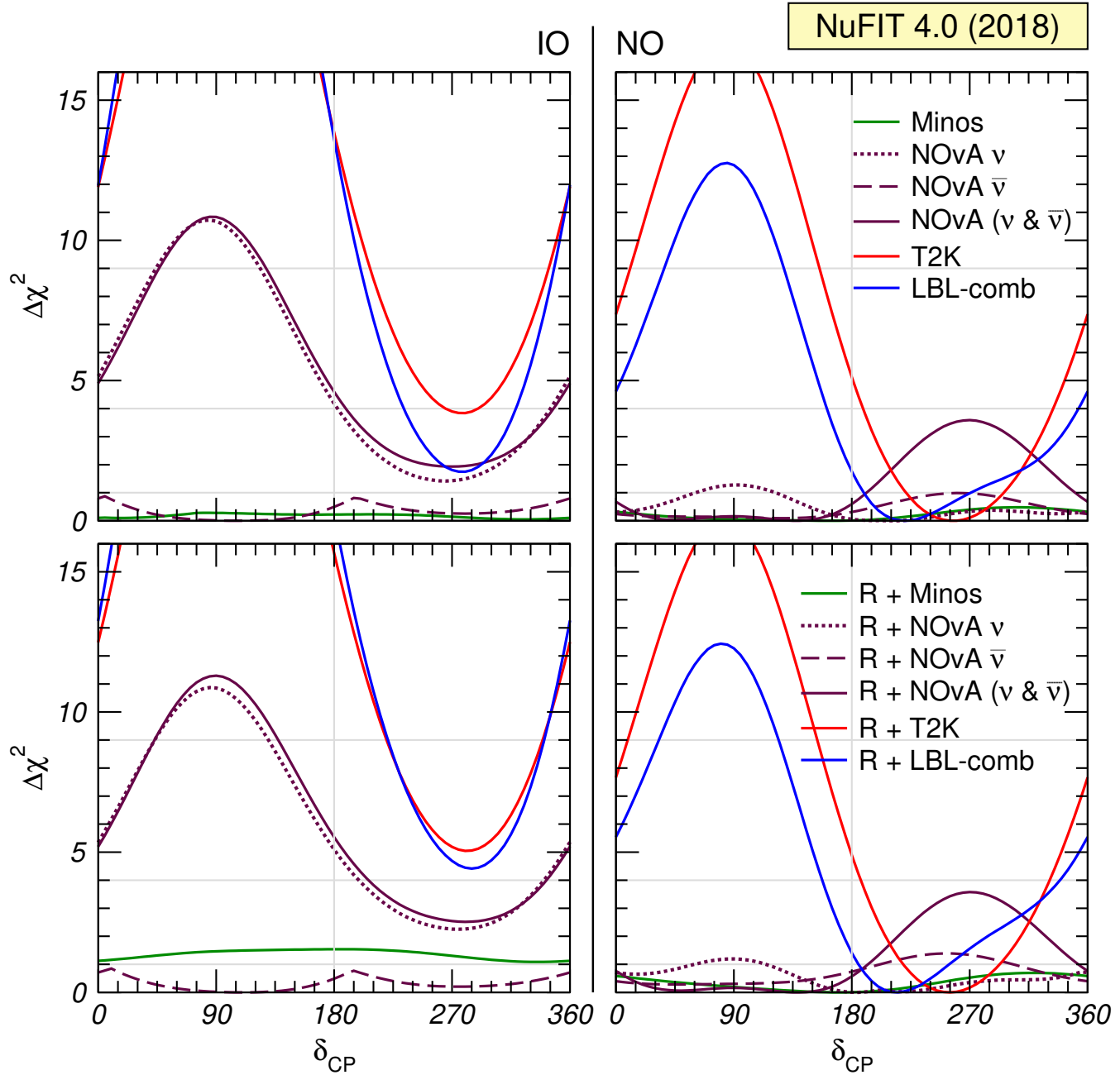

Figure 8. $\delta_{\mathrm{CP}}$ determination from LBL, reactor and their combination. Left (right) panels are for IO (NO). The upper panels show the 1-dim $\Delta \chi^{2}$ from LBL experiments after constraining only $\theta_{13}$ from reactor experiments. For each experiment $\Delta \chi^{2}$ is defined with respect to the global minimum of the two orderings. The lower panels show the corresponding determination when the full information of LBL accelerator and reactor experiments on both mixing angles and $\Delta m_{3 \ell}^{2}$ is used in the combination. In all panels $\Delta m_{21}^{2}, \sin ^{2} \theta_{12}$ are fixed to the global best fit values.

Let us first discuss the indication coming from LBL accelerator experiments. We find that $\mathrm{T} 2 \mathrm{~K}+$ the $\theta_{13}$ constraint from reactors disfavours IO by $\Delta \chi^{2} \approx 4$, see upper panels of figures 7,8 and 9 . This can be understood from the numbers in table 2 and eqs. (3.8) and (3.9), where the matter effect for NO helps to increase (decrease) events for neutrinos (anti-neutrinos). NOvA data + the $\theta_{13}$ constraint also disfavours IO by about 2 units in $\chi^{2}$, driven by neutrino data, while anti-neutrinos are insensitive to the ordering, cf. figure 8 . Interestingly, by combining T2K, NOvA, and MINOS, decreases the $\Delta \chi^{2}$ of IO to about 2. An explanation for this effect is the slight tension between NOvA and T2K in the determination of $\delta_{\mathrm{CP}}$ for $\mathrm{NO}$ visible in figure 8. This leads to a worse fit of NO compared to IO, where both experiments prefer the same region for $\delta_{\mathrm{CP}}$. 


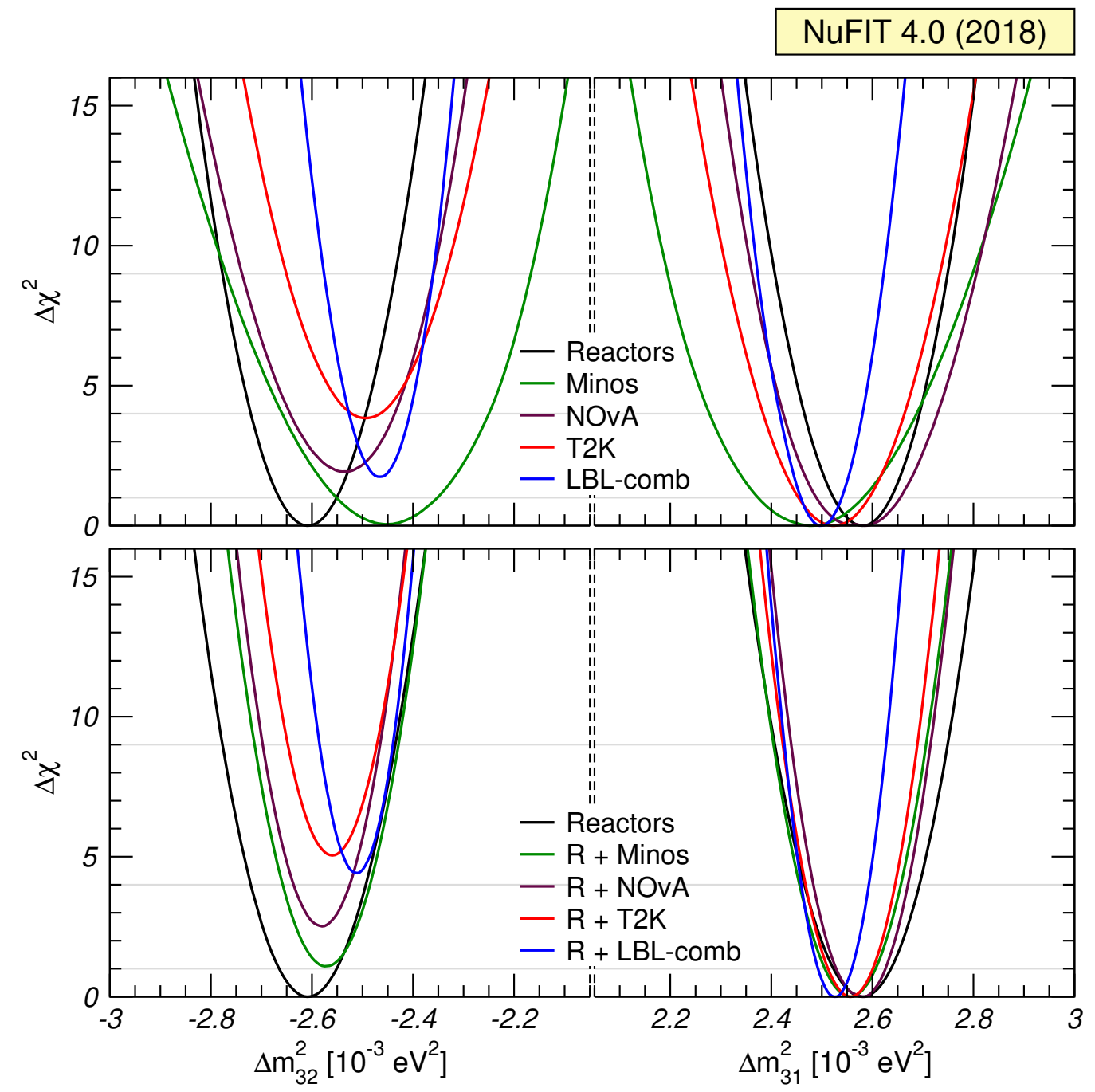

Figure 9. $\Delta m_{3 \ell}^{2}$ determination from LBL, reactor and their combination. Left (right) panels are for IO (NO). The upper panels show the 1-dim $\Delta \chi^{2}$ from LBL experiments after constraining only $\theta_{13}$ from reactor experiments. For each experiment $\Delta \chi^{2}$ is defined with respect to the global minimum of the two orderings. The lower panels show the corresponding determination when the full information of LBL accelerator and reactor experiments is used in the combination (including the information on $\Delta m_{3 \ell}^{2}$ from reactors). In all panels $\Delta m_{21}^{2}, \sin ^{2} \theta_{12}$ are fixed to the global best fit values.

An interesting additional effect sensitive to the mass ordering has been pointed out in refs. [44, 48]: the $\nu_{\mu}$ disappearance probability is symmetric with respect to the sign of $\Delta m_{\mu \mu}^{2}$ given in eq. (3.4), while $\nu_{e}$ disappearance is symmetric with respect to a slightly different effective mass-squared difference:

$$
\Delta m_{e e}^{2}=\cos ^{2} \theta_{12} \Delta m_{31}^{2}+\sin ^{2} \theta_{12} \Delta m_{32}^{2} .
$$

Hence, from a precise determination of the oscillation frequencies in $\nu_{\mu}$ and $\nu_{e}$ disappearance experiments, information on the sign of $\Delta m_{3 \ell}^{2}$ can be obtained. ${ }^{3}$ Indeed, we observe

\footnotetext{
${ }^{3} \mathrm{~A}$ similar effect has been exploited in ref. [49], based on the comparison of the $\Delta m_{3 \ell}^{2}$ determination in future reactor and atmospheric neutrino experiments.
} 
in figure 9 that this effect already contributes notably to the mass ordering discrimination in present data: the upper panels show the determination of $\Delta m_{3 \ell}^{2}$ from the individual LBL accelerator experiments $\left(\nu_{\mu}\right.$ disappearance) compared to the one from MBL reactors ( $\nu_{e}$ disappearance). We have verified that those curves are indeed symmetric with respect to the sign of $\Delta m_{\mu \mu}^{2}$ and $\Delta m_{e e}^{2}$, respectively, within excellent accuracy. When displaying them for common parameters $\left(\Delta m_{3 \ell}^{2}\right.$ in figure 9$)$, we observe that the agreement is better for NO than for IO. The difference between the upper and lower panels in the $\Delta \chi^{2}$ for IO is largely due to this $\Delta m_{3 \ell}^{2}$ effect. We see that the $\Delta \chi^{2}$ for the LBL combination is pushed from 2 to about 4.5, when combined consistently with reactor data taking into account the $\Delta m_{3 \ell}^{2}$ dependence.

In summary, we obtain from LBL+reactor data a preference for $\mathrm{NO}$ at about $2 \sigma$. As mentioned in section 2.2, this gets further enhanced by atmospheric neutrino data, with the main contribution from Super-Kamiokande, leading to the exclusion of IO at about $3 \sigma$, see figure 1. In the following subsection we discuss in more detail various aspects of the atmospheric neutrino analyses from IceCube and Super-Kamiokande.

\subsection{Treatment of atmospheric results from Super-Kamiokande and Deep-Core}

In what respects the atmospheric neutrino data, in our default analysis - full lines in figures 1 and 3 (one-dimensional $\Delta \chi^{2}$ curves) and coloured regions in figure 2 (twodimensional projections of confidence regions) - we include the results of the Deep-Core 3-years data of refs. [27, 28] (which we refer here as DC16) for which the collaboration has provided enough information on their effective areas to allow for our own reanalysis. Its impact in the parameter determination obtained from the combination of solar, reactor and LBL data is very marginal, see figure 10, which displays as example its contribution to the determination of $\Delta m_{3 \ell}^{2}$ and the ordering where it adds about 0.3 units to $\chi_{\min }^{2}$ of IO because of the slightly better matching between the $\Delta m_{3 \ell}^{2}$ from reactor+LBL experiments with that of DC in NO. ${ }^{4}$

In this respect it is interesting to notice that the ICECUBE collaboration has recently published the results of a dedicated analysis of another set of three-years data [31, 50] leading to a better determination of $\Delta m_{3 \ell}^{2}$ (which we refer to as DC17). Unfortunately we cannot reproduce this analysis because the corresponding effective areas have not been made public. The experiment has only made available the bi-dimensional $\chi^{2}$ map (as a function of $\Delta m_{3 \ell}^{2}$ and $\sin ^{2} \theta_{23}$ for a fixed value of $\sin ^{2} \theta_{13}=0.0217$ and $\delta_{\mathrm{CP}}=0$ ) corresponding to that analysis. Strictly this cannot be added in the global analysis without making some assumption about their possible $\theta_{13}$ and $\delta_{\mathrm{CP}}$ dependence. Still, to illustrate the possible impact of using these results we show also in figure 10 the corresponding contribution to the determination of $\Delta m_{3 \ell}^{2}$ and the ordering obtained by naively adding their $\chi^{2}$ map to our results of the global reactor+LBL experiments (neglecting any possible dependence on the fixed parameters). As seen in the figure, using the DC17 results in the global combination disfavours IO by $\sim 1.2$ additional units of $\chi^{2}$. One must notice,

\footnotetext{
${ }^{4}$ All curves in figure 10 contain the bias on $\Delta m_{21}^{2}$ and $\theta_{12}$ from solar and KamLAND, so the full lines denoted as $\mathrm{R}+\mathrm{LBL}+\mathrm{DC} 16$ coincide with the corresponding full lines in the corresponding panel in figure 1 .
} 


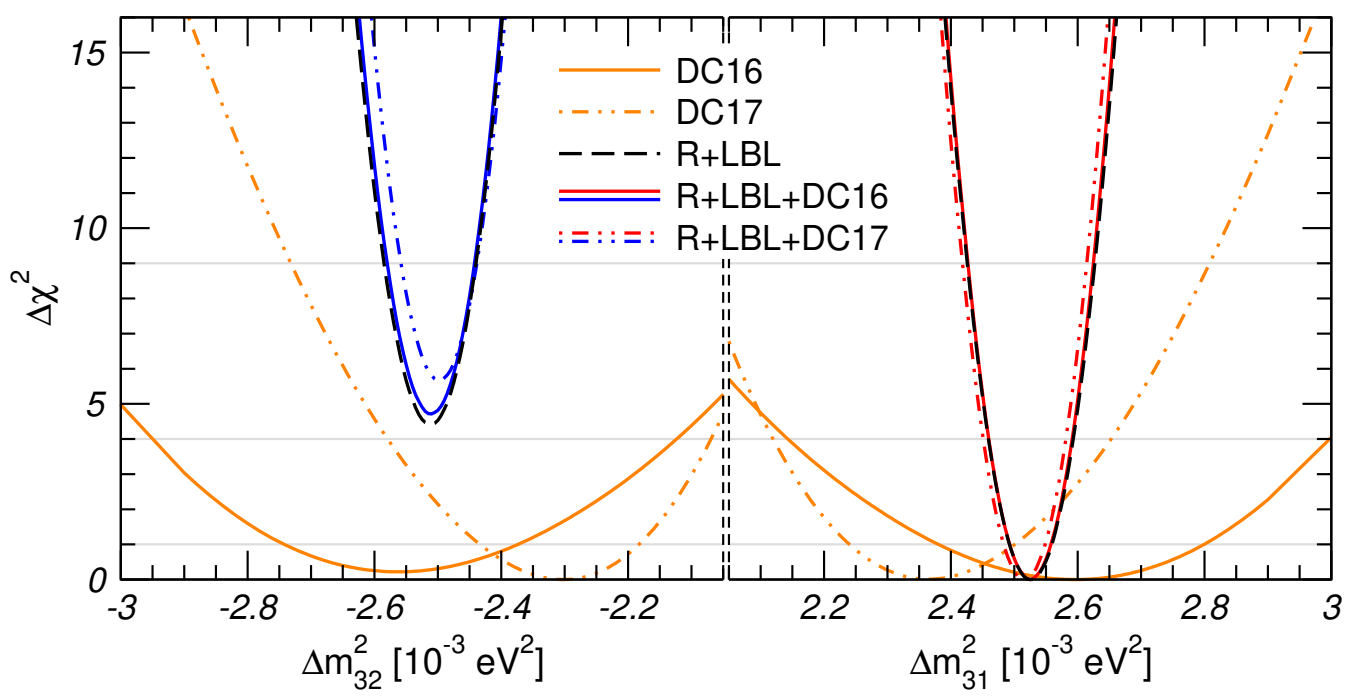

Figure 10. $\Delta \chi^{2}$ as a function of $\Delta m_{3 \ell}^{2}$ for our reanalysis of Deep-Core 3-years data of refs. [27, 28] (labeled DC16, solid orange line) and its combination with the global analysis of reactor and LBL experiments (full blue and red lines). The corresponding dash-dotted line correspond to use the $\chi^{2}$ table provided by the experiment for the analysis of their three years data in refs. [31, 50] (labeled DC17). See text for details.

however, that the ICECUBE collaboration has recently performed a reanalysis of the same data sample which leads to similar precision but a somewhat shifted range for $\Delta m_{3 \ell}^{2}[51]$.

In what respects to the results of Super-Kamiokande, in the last five years the collaboration has developed a more sophisticated analysis method for their atmospheric neutrino data with the aim of constructing $\nu_{e}+\bar{\nu}_{e}$ enriched samples which are then further classified into $\nu_{e}$-like and $\bar{\nu}_{e}$-like subsamples, thus increasing the sensitivity to subleading parameters such as the mass ordering and $\delta_{\mathrm{CP}}$. The official results obtained with this method were published in ref. [29] and show - once $\theta_{13}$ is constrained to be within the range determined by reactor experiments - a preference for $\mathrm{NO}$ with a $\Delta \chi^{2}(\mathrm{IO})=4.3$, variation of $\chi^{2}\left(\delta_{\mathrm{CP}}\right)$ with the $\mathrm{CP}$ phase at the level of $\sim 90 \% \mathrm{CL}$ (with favouring $\delta_{\mathrm{CP}} \sim 270^{\circ}$ ), and a slight favouring of the second octant of $\theta_{23}$ (see figure 14 in ref. [29]).

Unfortunately with the information at hand we are not able to reproduce the elements driving the main dependence on these subdominant oscillation effects in our own reanalysis of the data samples which can be simulated outside of the collaboration. However, SuperKamiokande has also published the results of their analysis in the form of a tabulated $\chi^{2}$ map [30] as a function of the four relevant parameters $\Delta m_{3 \ell}^{2}, \theta_{23}, \theta_{13}$, and $\delta_{\mathrm{CP}}$ which we can add to our global analysis $\chi^{2}$ in the multidimensional parameter space in a fully consistent form and then perform the corresponding parameter marginalization to obtained the combined one-dimensional or two dimensional parameter ranges. The results of such combination are shown as dashed curves in figures 1 and 3 (one-dimensional $\Delta \chi^{2}$ curves) and void regions in figure 2 (two-dimensional projections of confidence regions). As can be 
seen from figure 1, adding the SK-atm $\chi^{2}$ information results into:

- Increase of the $\chi_{\min }^{2}$ for IO by 4.6 units (from 4.7 to 9.3 ).

- Enhancement of the parameter dependence of $\chi^{2}\left(\delta_{\mathrm{CP}}\right)$ further disfavouring $\delta_{\mathrm{CP}}$ values around $90^{\circ}$ (for example it increases $\chi^{2}\left(\delta_{\mathrm{CP}}=70^{\circ}\right)$ in NO by $\sim 3$ units from $\sim 13$ to $\sim 16)$.

- Enhancement of the parameter dependence of $\chi^{2}\left(s_{23}^{2}\right)$ further disfavouring the first octant (for example it increases $\chi^{2}\left(s_{23}^{2}=0.45-0.5\right)$ in NO by $\sim 2$ units.

In other words, as the SK-atm tendencies for these subdominant effects are very well aligned with those from the combination of LBL experiments (currently dominated by T2K), their impact in the determination of $\delta_{\mathrm{CP}}$ and $\theta_{23}$ in the global analysis is almost equivalent to just adding for each of those parameters their marginalized $\chi^{2}$ (with fixed $\theta_{13}$ at the reactor value) to that from the global analysis without SK-atm.

\section{Projections on neutrino mass scale observables}

Oscillation experiments provide information on the mass-squared splittings $\Delta m_{i j}^{2}$ and on the leptonic mixing angles $U_{i j}$, but they are insensitive to the absolute mass scale for the neutrinos. Of course, the results of an oscillation experiment do provide a lower bound on the heavier mass in $\Delta m_{i j}^{2},\left|m_{i}\right| \geq \sqrt{\Delta m_{i j}^{2}}$ for $\Delta m_{i j}^{2}>0$, but there is no upper bound on this mass. In particular, the corresponding neutrinos could be approximately degenerate at a mass scale that is much higher than $\sqrt{\Delta m_{i j}^{2}}$. Moreover, there is neither an upper nor a lower bound on the lighter mass $m_{j}$.

Information on the neutrino masses, rather than mass differences, can be extracted from kinematic studies of reactions in which a neutrino or an anti-neutrino is involved. In the presence of mixing the most relevant constraint comes from the study of the end point $\left(E \sim E_{0}\right)$ of the electron spectrum in Tritium beta decay ${ }^{3} \mathrm{H} \rightarrow{ }^{3} \mathrm{He}+e^{-}+\bar{\nu}_{e}$. This spectrum can be effectively described by a single parameter, $m_{\nu_{e}}$, if for all neutrino states $E_{0}-E \gg m_{i}$ :

$$
\begin{aligned}
m_{\nu_{e}}^{2} & =\frac{\sum_{i} m_{i}^{2}\left|U_{e i}\right|^{2}}{\sum_{i}\left|U_{e i}\right|^{2}}=\sum_{i} m_{i}^{2}\left|U_{e i}\right|^{2}=c_{13}^{2} c_{12}^{2} m_{1}^{2}+c_{13}^{2} s_{12}^{2} m_{2}^{2}+s_{13}^{2} m_{3}^{2} \\
& = \begin{cases}\text { NO: } & m_{0}^{2}+\Delta m_{21}^{2} c_{13}^{2} s_{12}^{2}+\Delta m_{3 \ell}^{2} s_{13}^{2} \\
\text { IO: } & m_{0}^{2}-\Delta m_{21}^{2} c_{13}^{2} c_{12}^{2}-\Delta m_{3 \ell}^{2} c_{13}^{2}\end{cases}
\end{aligned}
$$

where the second equality holds if unitarity is assumed and $m_{0}=m_{1}\left(m_{3}\right)$ in NO (IO) denotes the lightest neutrino mass. At present we only have an upper bound, $m_{\nu_{e}} \leq 2.2 \mathrm{eV}$ at $95 \%$ CL [52], which is expected to be superseded soon by KATRIN [53] with about one order of magnitude improvement in sensitivity.

Direct information on neutrino masses can also be obtained from neutrinoless double beta decay $(A, Z) \rightarrow(A, Z+2)+e^{-}+e^{-}$. This process violates lepton number by two units, hence in order to induce the $0 \nu \beta \beta$ decay, neutrinos must be Majorana particles. In 

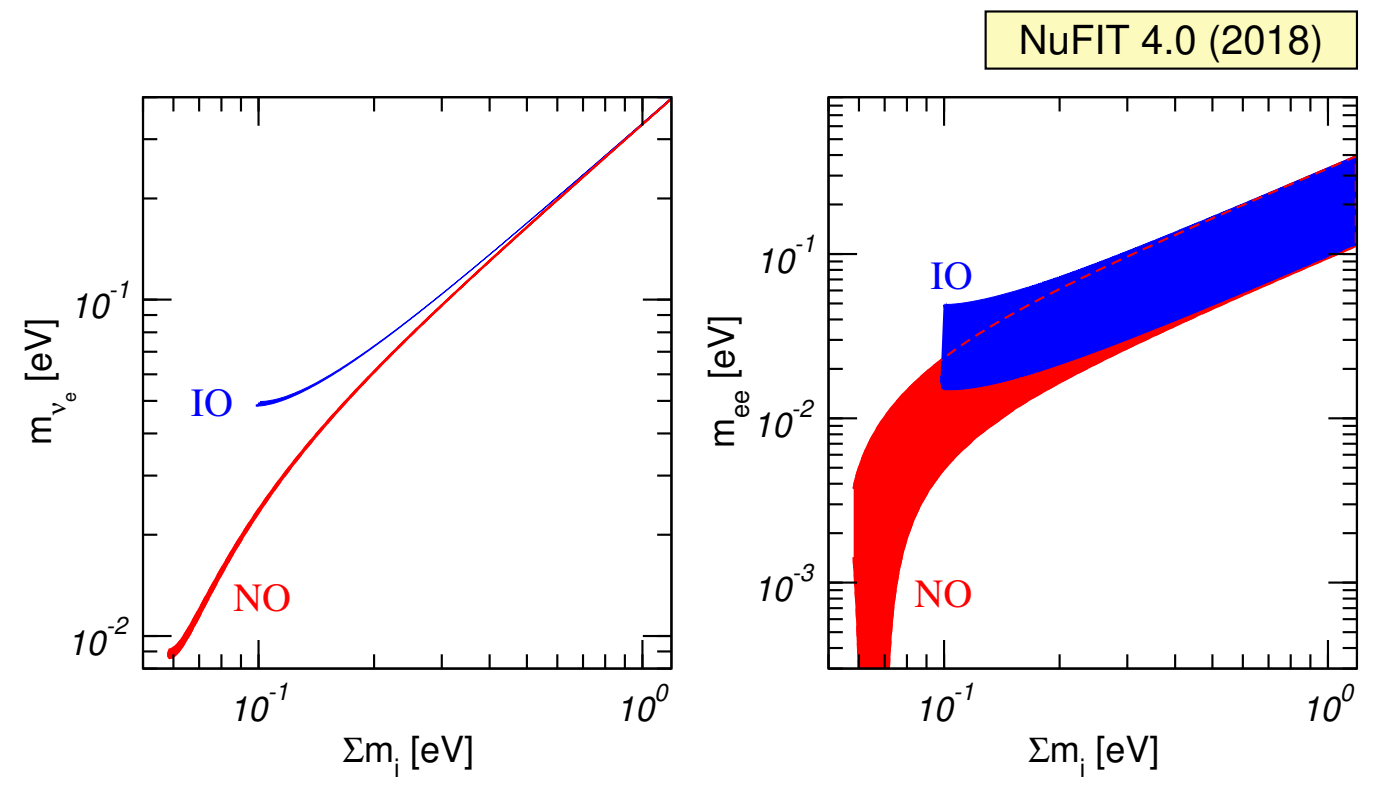

Figure 11. $95 \%$ allowed regions (for 2 dof) in the planes $\left(m_{\nu_{e}}, \sum m_{\nu}\right)$ and $\left(m_{e e}, \sum m_{\nu}\right)$ obtained from projecting the results of the global analysis of oscillation data. The regions are defined with respect to the minimum for each ordering.

particular, for the case in which the only effective lepton number violation at low energies is induced by the Majorana mass term for the neutrinos, the rate of $0 \nu \beta \beta$ decay is proportional to the effective Majorana mass of $\nu_{e}$ :

$$
\begin{aligned}
m_{e e} & =\left|\sum_{i} m_{i} U_{e i}^{2}\right|=\left|m_{1} c_{13}^{2} c_{12}^{2} e^{i 2 \alpha_{1}}+m_{2} c_{13}^{2} s_{12}^{2} e^{i 2 \alpha_{2}}+m_{3} s_{13}^{2} e^{-i 2 \delta_{\mathrm{CP}}}\right| \\
& = \begin{cases}\mathrm{NO}: & m_{0}\left|c_{13}^{2} c_{12}^{2} e^{i 2\left(\alpha_{1}-\delta_{\mathrm{CP}}\right)}+\sqrt{1+\frac{\Delta m_{21}^{2}}{m_{0}^{2}}} c_{13}^{2} s_{12}^{2} e^{i 2\left(\alpha_{2}-\delta_{\mathrm{CP}}\right)}+\sqrt{1+\frac{\Delta m_{3 \ell}^{2}}{m_{0}^{2}}} s_{13}^{2}\right| \\
\mathrm{IO}: & m_{0}\left|\sqrt{1-\frac{\Delta m_{3 \ell}^{2}+\Delta m_{21}^{2}}{m_{0}^{2}}} c_{13}^{2} c_{12}^{2} e^{i 2\left(\alpha_{1}-\delta_{\mathrm{CP}}\right)}+\sqrt{1-\frac{\Delta m_{3 \ell}^{2}}{m_{0}^{2}}} c_{13}^{2} s_{12}^{2} e^{i 2\left(\alpha_{2}-\delta_{\mathrm{CP}}\right)}+s_{13}^{2}\right|\end{cases}
\end{aligned}
$$

which, unlike eq. (4.1), depends also on the $\mathrm{CP}$ violating phases. Recent searches have established the lifetime of this decay to be longer than $\sim 10^{26}$ yr $[54,55]$, corresponding to a limit on the neutrino mass of $m_{e e} \lesssim 0.06-0.200 \mathrm{eV}$ at $90 \%$ CL. A series of new experiments is planned with sensitivity of up to $m_{e e} \sim 0.01 \mathrm{eV}[56]$.

Neutrino masses have also interesting cosmological effects. In general, cosmological data mostly give information on the sum of the neutrino masses, $\sum_{i} m_{i}$, while they have very little to say on their mixing structure and on the ordering of the mass states.

Correlated information on these three probes of the neutrino mass scale can be obtained by mapping the results from the global analysis of oscillations presented previously. We show in figure 11 the present status of this exercise. The relatively large width of the regions in the right panel are due to the unknown Majorana phases. Thus from a positive determination of two of these probes, in principle information can be obtained on the value of the Majorana phases and/or the mass ordering [57, 58]. 


\section{Conclusions}

We have presented the results of the updated (as of fall 2018) analysis of relevant neutrino data in the framework of mixing among three massive neutrinos. We have shown our results for two analyses. The first contains our own statistical combination of all the experimental data for which we are able to reproduce the results of the partial analysis performed by the different experiments, and therefore does not include the information of the Super-Kamiokande atmospheric neutrino data. In the second analysis we combine the likelihood of the first one with the four-dimensional $\chi^{2}$ map provided by Super-Kamiokande for the analysis of their atmospheric data. Quantitatively the present determination of the two mass differences, three mixing angles and the relevant $\mathrm{CP}$ violating phase for the two analysis is listed in table 1, and the corresponding leptonic mixing matrix is given in eq. (2.2). In both analysis the maximum allowed $\mathrm{CP}$ violation in the leptonic sector parametrized by the Jarlskog determinant is $J_{\mathrm{CP}}^{\max }=0.0333 \pm 0.0006( \pm 0.0019)$ at $1 \sigma(3 \sigma)$.

We have performed a detail study of the role of the different data samples and their correct combination in the determination of the less known parameters, $\theta_{23}, \delta_{\mathrm{CP}}$ and the ordering in section 3 . We can summarize the main conclusions in this section as follows:

- The long standing tension between the best $\Delta m_{21}^{2}$ determined in the solar neutrino analysis and that from KamLAND persists. The inclusion of latest spectral data from SK4 and the use of Daya Bay near detector data for reactor flux normalization in KamLAND has made this tension slightly stronger, but it is still a $\sim 2 \sigma$ effect.

- We obtain preference for the second octant of $\theta_{23}$ in the global analysis with a best fit at $\sin ^{2} \theta_{23}=0.58$. There are two effects contributing to this results:

- While most data samples in $\nu_{\mu}$ and $\bar{\nu}_{\mu}$ disappearance at LBL prefer close to maximal mixing (especially $\mathrm{T} 2 \mathrm{~K}$ and NOvA neutrino data), maximal mixing is disfavoured by MINOS neutrino data $\left(\Delta \chi^{2} \approx 2\right)$ and NOvA anti-neutrino data $\left(\Delta \chi^{2} \approx 6\right)$. This disfavouring increases when fully combining with the reactor neutrino determination of $\Delta m_{3 \ell}^{2}$ to $\Delta \chi^{2} \approx 7$ and 9 , respectively.

- The appearance results both in T2K and NOvA (SK-atm adds in the same direction) favour the second octant. The final value of the best fit $\theta_{23}$ results of this effect in combination with the substantial non-maximality favoured by NOvA anti-neutrino and MINOS neutrino disappearance data.

- The determination of $\delta_{\mathrm{CP}}$ is mostly driven by T2K neutrino and anti-neutrino appearance results which favour $\delta_{\mathrm{CP}} \sim 3 \pi / 2$ and disfavours $\delta_{\mathrm{CP}} \sim \pi / 2$ for both NO and IO. NOvA neutrino appearance data align with this behaviour, and are more statistically significant in IO. On the contrary NOvA anti-neutrino appearance data are better (worse) described with $\delta_{\mathrm{CP}} \sim \pi / 2\left(\delta_{\mathrm{CP}} \sim 3 \pi / 2\right)$ in NO. This slight tension results into a shift of the best fit to $\delta_{\mathrm{CP}}=215^{\circ}$ in NO. So the allowed range is pushed towards the $\mathrm{CP}$ conserving value of $180^{\circ}$, which now is only disfavoured with $\Delta \chi^{2} \lesssim 2$ 
- Regarding the mass ordering:

- Both T2K and NOvA prefer NO individually: T2K (NOvA) + the $\theta_{13}$ constraint from reactors disfavours IO by $\Delta \chi^{2} \approx 4(2)$, but combining T2K, NOvA (and MINOS) decreases the $\Delta \chi^{2}$ of IO to about 2 . This is a consequence of the slight tension between NOvA and $\mathrm{T} 2 \mathrm{~K}$ in the determination of $\delta_{\mathrm{CP}}$ for $\mathrm{NO}$.

- Additional sensitivity to the ordering is found by the precise determination of the oscillation frequency in $\nu_{\mu}$ and $\nu_{e}$ disappearance data at LBL and reactors, respectively. This effect increases $\Delta \chi^{2}$ of IO from 2 to about 4.5 .

- Inclusion of the atmospheric neutrino results (mainly from SK) further increases $\Delta \chi^{2}$ of IO to the $3 \sigma$ level.

Future updates of this analysis will be provided at the NuFIT website quoted in ref. [12].

\section{Acknowledgments}

This work is supported by USA-NSF grant PHY-1620628, by EU Networks FP10 ITN ELUSIVES (H2020-MSCA-ITN-2015-674896) and INVISIBLES-PLUS (H2020-MSCA-RISE2015-690575), by MINECO grant FPA2016-76005-C2-1-P and MINECO/FEDER-UE grants FPA2015-65929-P and FPA2016-78645-P, by Maria de Maetzu program grant MDM2014-0367 of ICCUB, and by the "Severo Ochoa" program grant SEV-2016-0597 of IFT. I.E. acknowledges support from the FPU program fellowship FPU15/03697.

\section{A List of data used in the analysis}

\section{Solar experiments}

- External information: Standard Solar Model [38].

- Chlorine total rate [59], 1 data point.

- Gallex \& GNO total rates [60], 2 data points.

- SAGE total rate [61], 1 data point.

- SK1 full energy and zenith spectrum [62], 44 data points.

- SK2 full energy and day/night spectrum [63], 33 data points.

- SK3 full energy and day/night spectrum [64], 42 data points.

- SK4 2055-day day-night asymmetry [42] and 2860-day energy spectrum [41], 24 data points.

- SNO combined analysis [65], 7 data points.

- Borexino Phase-I 741-day low-energy data [66], 33 data points.

- Borexino Phase-I 246-day high-energy data [67], 6 data points.

- Borexino Phase-II 408-day low-energy data [68], 42 data points. 


\section{Atmospheric experiments}

- External information: Atmospheric neutrino fluxes [69].

- IceCube/DeepCore 3-year data [27, 28], 64 data points.

- SK1-4 328 kiloton years [29], $\chi^{2}$ map [30] added to our global analysis.

\section{Reactor experiments}

- KamLAND separate DS1, DS2, DS3 spectra [70] with Daya Bay reactor $\nu$ fluxes [25], 69 data points.

- Double Chooz FD-I/ND and FD-II/ND spectral ratios, with 455-day (FD-I), 363-day (FD-II) and 258-day (ND) exposures [71], 56 data points.

- Daya Bay 1958-day EH2/EH1 and EH3/EH1 spectral ratios [21], 52 data points.

- Reno 2200-day FD/ND spectral ratios [22], 26 data points.

\section{Accelerator experiments}

- MINOS $10.71 \times 10^{20}$ pot $\nu_{\mu}$-disappearance data [15], 39 data points.

- MINOS $3.36 \times 10^{20}$ pot $\bar{\nu}_{\mu}$-disappearance data [15], 14 data points.

- MINOS $10.6 \times 10^{20}$ pot $\nu_{e}$-appearance data [16], 5 data points.

- MINOS $3.3 \times 10^{20}$ pot $\bar{\nu}_{e}$-appearance data [16], 5 data points.

- T2K $14.93 \times 10^{20}$ pot $\nu_{\mu}$-disappearance data [72], 55 data points.

- T2K $14.93 \times 10^{20}$ pot $\nu_{e}$-appearance data [72], 23 data points for the CCQE and 16 data points for the $\mathrm{CC} 1 \pi$ samples.

- T2K $11.24 \times 10^{20}$ pot $\bar{\nu}_{\mu}$-disappearance data [73], 55 data points.

- $\mathrm{T} 2 \mathrm{~K} 11.24 \times 10^{20}$ pot $\bar{\nu}_{e}$-appearance data [73], 23 data points.

- NOvA $8.85 \times 10^{20}$ pot $\nu_{\mu}$-disappearance data [46], 76 data points.

- NOvA $8.85 \times 10^{20}$ pot $\nu_{e}$-appearance data [46], 13 data points.

- NOvA $6.91 \times 10^{20}$ pot $\bar{\nu}_{\mu}$-disappearance data [46], 76 data points.

- NOvA $6.91 \times 10^{20}$ pot $\bar{\nu}_{e}$-appearance data [46], 12 data points.

\section{B Technical details and validation cross checks}

This this appendix we provide some details on our analysis of the most recent data from accelerator and reactor experiments, and show that we can reproduce the results of the experimental collaborations with good accuracy, when using the same assumptions in the analysis. 


\section{B.1 T2K}

The predicted number of events in the T2K far detector in a given energy bin $i$ and for a given channel $\alpha$ can be calculated as

$$
N_{i}^{\alpha}=N_{\mathrm{bkg}, \mathrm{i}}+\int_{E_{i}}^{E_{i+1}} \mathrm{~d} E_{\mathrm{rec}} \int_{0}^{\infty} \mathrm{d} E_{\nu} R\left(E_{\mathrm{rec}}, E_{\nu}\right) \frac{\mathrm{d} \Phi}{\mathrm{d} E_{\nu}} \sigma_{\alpha}\left(E_{\nu}\right) \varepsilon\left(E_{\nu}\right) P_{\nu_{\mu} \rightarrow \nu_{\alpha}}\left(E_{\nu}\right),
$$

where

- $N_{\mathrm{bkg}, \mathrm{i}}$ is the number of background events in that bin, which we have extracted from ref. [72] and consistently re-scaled to the latest exposure. If there is a neutrino component, its oscillation has to be consistently included.

- $\left[E_{i}, E_{i+1}\right]$ are the bin limits.

- $E_{\text {rec }}$ is the reconstructed neutrino energy.

- $E_{\nu}$ is the true neutrino energy.

- $R\left(E_{\mathrm{rec}}, E_{\nu}\right)$ is the energy reconstruction function, that we take to be Gaussian.

- $\frac{\mathrm{d} \Phi}{\mathrm{d} E_{\nu}}$ is the incident $\nu_{\mu}$ flux, extracted from ref. [74].

- $\sigma_{\alpha}$ is the $\nu_{\alpha}$ detector cross-section, extracted from ref. [74].

- $\varepsilon$ is the detection efficiency, which is adjusted to reproduce the observed spectra in ref. [73].

- $P_{\nu_{\mu} \rightarrow \nu_{\alpha}}\left(E_{\nu}\right)$ is the $\nu_{\mu} \rightarrow \nu_{\alpha}$ oscillation probability.

For the antineutrino channel, one has to switch $\nu$ by $\bar{\nu}$.

If we assume a Poissonian $\chi^{2}$ with the data points in ref. [73], add an overall normalisation systematic uncertainty ${ }^{5}$ and combine all the data, we get the contours in figure 12 . The other oscillation parameters are fixed as specified in ref. [73] and the reactor uncertainty on $\theta_{13}$ is included as a Gaussian bias and marginalised over. Finally, following the ad-hoc procedure described in section 8.4.2 in ref. [73], the disappearance $\Delta m_{32}^{2}$ contours are manually Gaussian-smeared with a standard deviation $\sigma=4.1 \cdot 10^{-5} \mathrm{eV}^{2}$.

\section{B.2 NOvA}

For NOvA the predicted number of events is given, as for T2K, by eq. (B.1). Fluxes and detector response are extracted from refs. [75, 76]. We take the data points and backgrounds from ref. [76] and with those construct a Poissonian $\chi^{2}$ including an normalisation systematic uncertainty ${ }^{6}$ and we get the contours in figure 13 when the undisplayed oscillation parameters are fixed as specified in ref. [76]. In particular the reactor uncertainty on $\theta_{13}$ is included as a Gaussian bias and marginalised over.

\footnotetext{
${ }^{5}$ We take it to be $7 \%$ for the $\nu_{\mu}$ and $\bar{\nu}_{\mu}$ disappearance channels, $5 \%$ for the $\bar{\nu}_{e}$ appearance channel, $8 \%$ for the CCQE $\nu_{e}$ appearance channel, and $23 \%$ for the CC1 $\pi \nu_{e}$ appearance channel.

${ }^{6}$ We take it to be $5 \%$ for $\nu_{e}$ appearance, $6 \%$ for $\bar{\nu}_{e}$ appearance, and $6 \%$ for both the $\nu_{\mu}$ and $\bar{\nu}_{\mu}$ disappearance channels. We take these uncertainties as fully correlated for the disappearance chanels.
} 

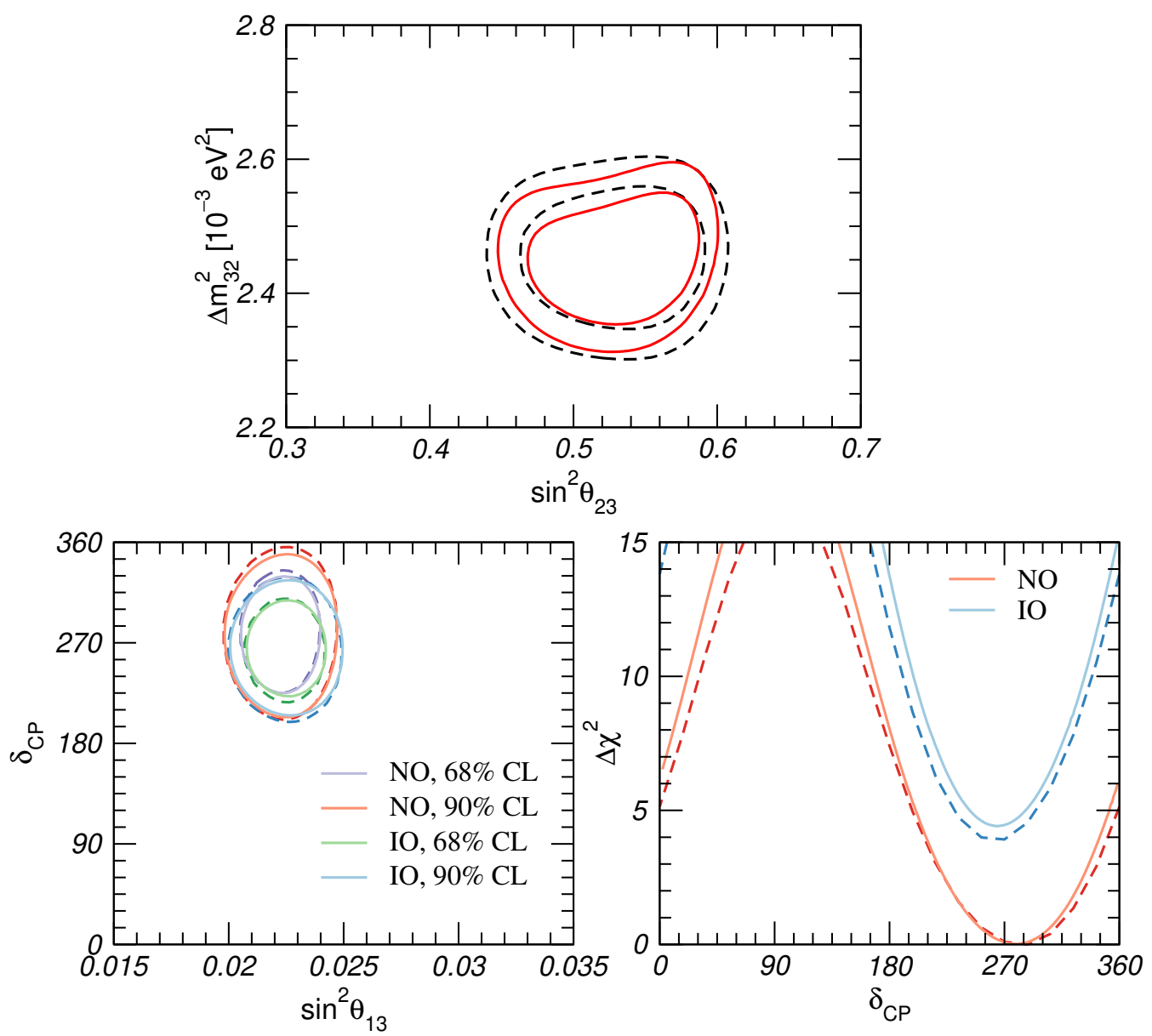

Figure 12. Different projections to our fit to latest T2K data (dashed lines) compared to the corresponding results of the experimental collaboration (solid curves), as presented in ref. [73], when adopting the same assumptions. All unshown parameters are marginalised over.

\section{B.3 Daya Bay}

The Daya Bay $3 \nu$ oscillation analysis is based on [21], the data is taken from the supplementary material provided on arXiv.

In each experimental hall in Daya Bay there are more than one detector, so the number of events in hall $H, N_{i}^{H}$ is a sum of the contributions in all the detectors in the hall. The predicted numbers of events in a detector $d$ in an energy bin $i$ is computed as

$$
N_{i}^{d}=\mathcal{N} \sum_{r} \sum_{\text {iso }} \frac{\epsilon^{d}}{L_{r d}^{2}} \int_{E_{i}^{\text {rec }}}^{E_{i+1}^{r e c}} d E^{\text {rec }} \int_{0}^{\infty} d E_{\nu} \sigma\left(E_{\nu}\right) f^{\text {iso }} \phi^{\text {iso }}\left(E_{\nu}\right) P_{\bar{\nu}_{e} \rightarrow \bar{\nu}_{e}}^{r d}\left(E_{\nu}\right) R\left(E^{\text {rec }}, E_{\nu}\right) .
$$

The indices $i, r, d$, iso refer to the energy bin, reactor, detector, and fissible isotope, respectively. $\epsilon^{d}$ are the detector efficiencies, including $\varepsilon_{\mu} \times \varepsilon_{m}$ multiplied by the life time days (taken both from table I in [21]) as well as the relative difference of target protons $\Delta N_{p}$ in each detector, obtained from table VI in [77]. $L_{r d}$ are the baselines between the reactor $r$ and detector $d$, obtained from table I in [77]. $E_{\nu}$ and $E^{\text {rec }}$ are the true and reconstructed 

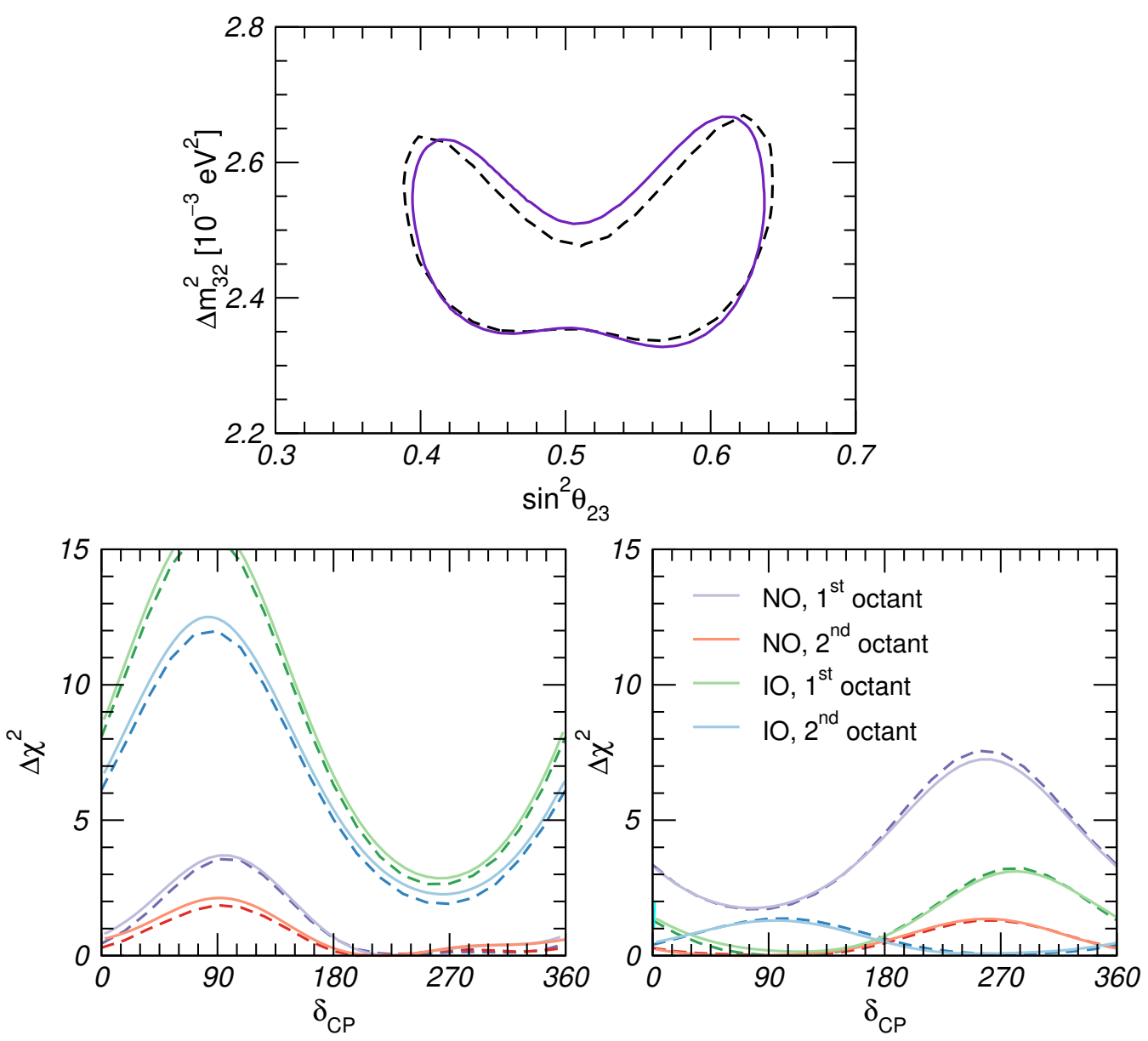

Figure 13. Different projections to our fit to latest NOvA data (dashed lines) compared to the corresponding results of the experimental collaboration (solid curves), as presented in ref. [46], when adopting the same assumptions. The upper plot only includes disappearance data, whereas the bottom left plot corresponds to $\nu_{e}$ appearance and the bottom right plot to $\bar{\nu}_{e}$ appearance.

neutrino energy, which are related by the detector response function $R\left(E^{\text {rec }}, E_{\text {prompt }}\right)$, provided in the complementary material to [21]. The relation between the prompt energy $E_{\text {prompt }}$ and the neutrino energy $E_{\nu}$ is $E_{\nu}=m_{n}-m_{p}-m_{e}+E_{\text {prompt }} . \sigma\left(E_{\nu}\right)$ is the Inverse Beta Decay cross section computed performing the integral over $\cos \theta$ of the differential cross section in [78]. $\phi^{\text {iso }}\left(E_{\nu}\right)$ are the Huber-Mueller flux predictions $[79,80]$ and $f^{\text {iso }}$ are the fission fractions. For each isotope, $f^{\text {iso }}$ is computed as the average of the fission fractions in table 9 of ref. [25]. Following section 2.6 of [25], we apply non-equilibrium corrections by adding the relative correction from table VII of [80] to the fluxes [79, 80]. Since Daya Bay has run for a long period we take the row corresponding to 450 days. $P_{\bar{\nu}_{e} \rightarrow \bar{\nu}_{e}}^{r d}\left(E_{\nu}\right)$ is the oscillation probability. The global constant $\mathcal{N}$ will cancel when taking ratios of event numbers. 
Our DayaBay $\chi^{2}$ is based on the ratios of the observed spectra in experimental halls 3 and 1 as well as 2 and 1 :

$$
\begin{aligned}
& \chi^{2}\left(\theta_{12}, \theta_{13}, \Delta m_{21}^{2}, \Delta\right.\left.m_{31}^{2}, \vec{\eta}\right)=\sum_{i} \frac{\left(\frac{O_{i}^{3}-B_{i}^{3}(\vec{\eta})}{O_{i}^{1}-B_{i}^{1}(\vec{\eta})}-\frac{N_{i}^{3}}{N_{i}^{1}}\left(\theta_{12}, \theta_{13}, \Delta m_{21}^{2}, \Delta m_{31}^{2}, \vec{\eta}\right)\right)^{2}}{\left(\sigma_{i}^{s t a t}\right)_{31}^{2}} \\
&+\sum_{i} \frac{\left(\frac{O_{i}^{2}-B_{i}^{2}(\vec{\eta})}{O_{i}^{1}-B_{i}^{1}(\vec{\eta})}-\frac{N_{i}^{2}}{N_{i}^{1}}\left(\theta_{12}, \theta_{13}, \Delta m_{21}^{2}, \Delta m_{31}^{2}, \vec{\eta}\right)\right)^{2}}{\left(\sigma_{i}^{\text {stat }}\right)^{2}}+\vec{\eta}^{T} V_{\eta}^{-1} \vec{\eta} .
\end{aligned}
$$

Here, $O_{i}^{H}$ and $B_{i}^{H}(\vec{\eta})$ are the observed number of events and the background predictions in the experimental hall $H$ and bin $i$, which can be found in the complementary material of [21]. $\sigma_{i}^{\text {stat }_{H H^{\prime}}}$ are the errors of the ratio $\left[O_{i}^{H}-B_{i}^{H}(\vec{\eta})\right] /\left[O_{i}^{H^{\prime}}-B_{i}^{H^{\prime}}(\vec{\eta})\right]$, computed propagating the statistical errors of $O_{i}^{H}$ and $O_{i}^{H^{\prime}}{ }^{7}$

In eq. (B.3), $\vec{\eta}$ is the vector of pull parameters and $V_{\eta}$ is the pull correlation matrix which accounts for the systematic uncertainties and their correlations. In order to reproduce the Daya Bay results [21], systematics in detection efficiency, relative energy scale and cosmogenic Li-He background have to be taken into account. The detection efficiency and relative energy scale uncertainties are given by $0.13 \%$ and $0.2 \%$, respectively [21, 77], and the Li-He background uncertainty is given by $30 \%$ [21]. We include also the uncertainties in accidental (1\%) and fast neutron (13\% (17\%) in EH1 and EH2 (EH3)) backgrounds [77], which however play only a subleading role. We take into account the 5 systematics in each of the 3 experimental hall as uncorrelated, so $V_{\eta}$ is a diagonal $15 \times 15$ matrix. In order to use the correct uncertainties in each experimental hall we divide the detector uncertainties by $\sqrt{2}$ in $\mathrm{EH} 1$ and $\mathrm{EH} 2$ and by $\sqrt{4}$ in $\mathrm{EH} 3$, since there are 2 and 4 detectors, respectively

In the left panel of figure 14, our re-analysis is compared to the one published in [21].

\section{B.4 RENO}

The RENO $3 \nu$ oscillation analysis is based on [22]. The number of events in the near and far detectors are computed as in eq. (B.2), using the Daya Bay response function. The average fission fractions are taken from [81], the baselines from [82], and life time days can be found in [22]. In order to compute the total relative efficiency between the near and far detectors, a normalization to the total number of predicted events without oscillations in the far detector is performed. The RENO $\chi^{2}$ is based on the far/near spectral ratio is implemented as follows:

$$
\begin{aligned}
& \chi^{2}\left(\theta_{12}, \theta_{13}, \Delta m_{21}^{2}, \Delta m_{31}^{2}, \vec{\eta}\right) \\
& \quad=\sum_{i} \frac{\left(\frac{O_{i}^{F}}{O_{i}^{N}}-\frac{N_{i}^{F}}{N_{i}^{N}}\left(\theta_{12}, \theta_{13}, \Delta m_{21}^{2}, \Delta m_{31}^{2}, \vec{\eta}\right)\right)^{2}}{\left(\sigma_{i}^{\text {stat }}\right)^{2}}+\vec{\eta}^{T} V_{\eta}^{-1} \vec{\eta} .
\end{aligned}
$$

\footnotetext{
${ }^{7}$ We neglect the correlation of the statistical errors due to the events in EH1, which appear in both ratios. In this way we obtain better agreement with the results of the DayaBay collaboration, cf. figure 14, indicating that this choice of errors provides a fair approximation.
} 

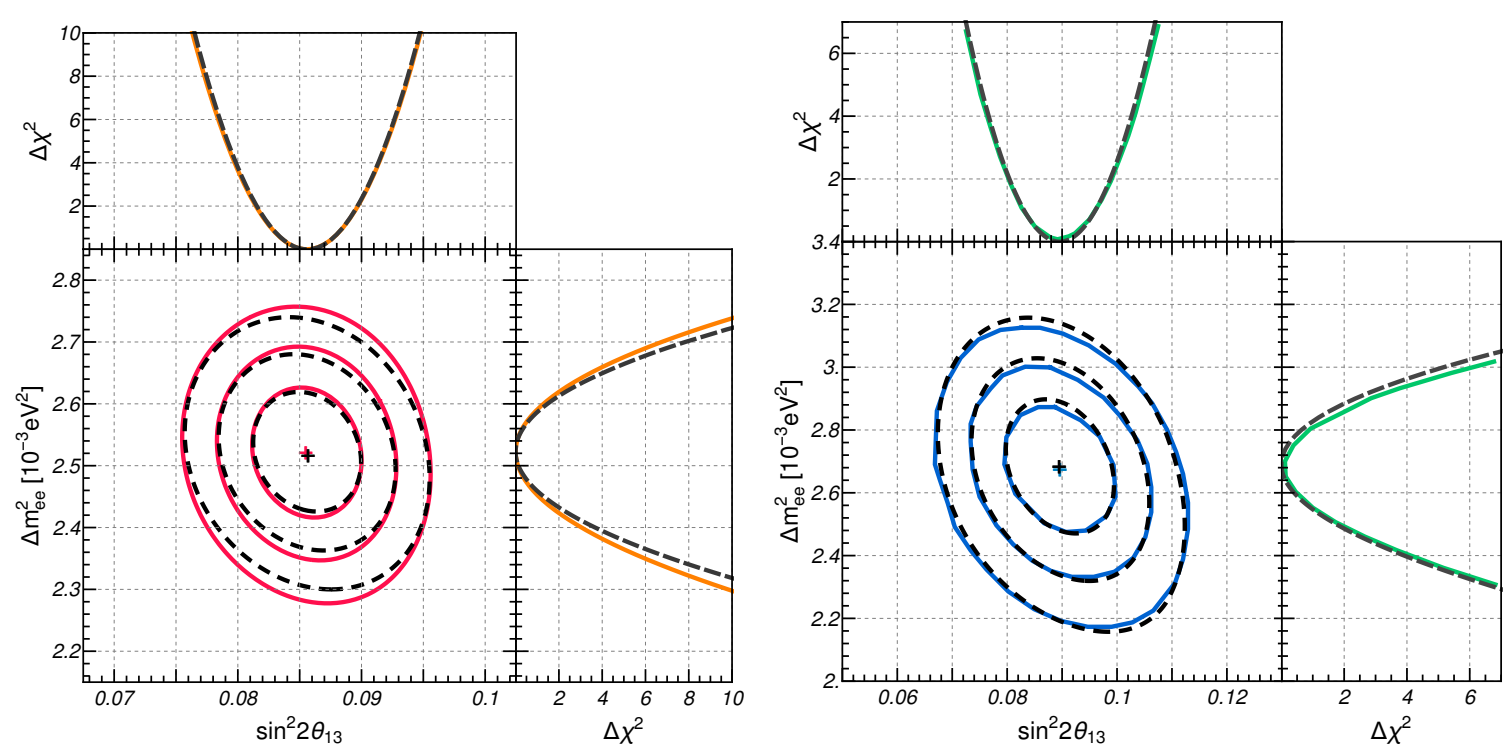

Figure 14. Our fit to DayaBay and RENO (black-dashed lines) compared to the results of the experimental collaborations (solid lines), as published in refs. [21] and [22], respectively, when adopting the same assumptions. Following the collaborations, on the vertical axes, the parameter $\Delta m_{e e}^{2}$ defined in eq. (3.10) is used.

Here, $O_{i}^{F / N}$, are the observed number of events in the far and near detectors and energy bin $i$, with background expectations subtracted. The data is obtained from digitizing figure 1 of [22]. $N_{i}^{F / N}$, are the corresponding predicted number of events, computed as in eq. (B.2) and $\sigma_{i}^{\text {stat }}$, are the statistical errors of the ratio $\frac{O_{i}^{F}}{O_{i}^{N}} \cdot \vec{\eta}$ is the vector of the pull parameters and $V_{\eta}$ is the pull correlation matrix which accounts for the systematic uncertainties associated to each pull parameter and their correlations.

Ref. [22] quotes systematic uncertainties in the relative detection efficiency and relative energy scale of $0.21 \%$ and $0.15 \%$, respectively. The cosmogenic Li-He background plays no significant role, but is included as well with a relative uncertainty of $5 \%(8 \%)$ for the near (far) detector, cf. table I of [22]. In order to match precisely the results shown in figure 3 of [22], an extra factor of 0.984 to the Far/Near ratio has to be included and the relative detection efficiency uncertainty has to be increased by a factor of 1.4. Our re-analysis of RENO data is compared to the official one in the right panel of figure 14.

Open Access. This article is distributed under the terms of the Creative Commons Attribution License (CC-BY 4.0), which permits any use, distribution and reproduction in any medium, provided the original author(s) and source are credited.

\section{References}

[1] B. Pontecorvo, Neutrino Experiments and the Problem of Conservation of Leptonic Charge, Sov. Phys. JETP 26 (1968) 984 [INSPIRE].

[2] V.N. Gribov and B. Pontecorvo, Neutrino astronomy and lepton charge, Phys. Lett. B 28 (1969) 493 [INSPIRE]. 
[3] Z. Maki, M. Nakagawa and S. Sakata, Remarks on the unified model of elementary particles, Prog. Theor. Phys. 28 (1962) 870 [InSPIRE].

[4] M. Kobayashi and T. Maskawa, CP Violation in the Renormalizable Theory of Weak Interaction, Prog. Theor. Phys. 49 (1973) 652 [InSPIRE].

[5] N. Cabibbo, Time Reversal Violation in Neutrino Oscillation, Phys. Lett. B 72 (1978) 333 [INSPIRE].

[6] S.M. Bilenky, J. Hosek and S.T. Petcov, On Oscillations of Neutrinos with Dirac and Majorana Masses, Phys. Lett. B 94 (1980) 495 [INSPIRE].

[7] V.D. Barger, K. Whisnant and R.J.N. Phillips, CP Violation in Three Neutrino Oscillations, Phys. Rev. Lett. 45 (1980) 2084 [INSPIRE].

[8] P. Langacker, S.T. Petcov, G. Steigman and S. Toshev, On the Mikheev-Smirnov-Wolfenstein (MSW) Mechanism of Amplification of Neutrino Oscillations in Matter, Nucl. Phys. B 282 (1987) 589 [INSPIRE].

[9] M.C. Gonzalez-Garcia, M. Maltoni and T. Schwetz, Updated fit to three neutrino mixing: status of leptonic CP-violation, JHEP 11 (2014) 052 [arXiv: 1409.5439] [INSPIRE].

[10] M.C. Gonzalez-Garcia, M. Maltoni, J. Salvado and T. Schwetz, Global fit to three neutrino mixing: critical look at present precision, JHEP 12 (2012) 123 [arXiv: 1209.3023] [INSPIRE].

[11] I. Esteban, M.C. Gonzalez-Garcia, M. Maltoni, I. Martinez-Soler and T. Schwetz, Updated fit to three neutrino mixing: exploring the accelerator-reactor complementarity, JHEP 01 (2017) 087 [arXiv: 1611.01514] [INSPIRE].

[12] Nufit webpage, http://www.nu-fit.org.

[13] P.F. de Salas, D.V. Forero, C.A. Ternes, M. Tortola and J.W.F. Valle, Status of neutrino oscillations 2018: $3 \sigma$ hint for normal mass ordering and improved CP sensitivity, Phys. Lett. B 782 (2018) 633 [arXiv:1708.01186] [InSPIRE].

[14] F. Capozzi, E. Lisi, A. Marrone and A. Palazzo, Current unknowns in the three neutrino framework, Prog. Part. Nucl. Phys. 102 (2018) 48 [arXiv:1804.09678] [INSPIRE].

[15] MINOS collaboration, Measurement of Neutrino and Antineutrino Oscillations Using Beam and Atmospheric Data in MINOS, Phys. Rev. Lett. 110 (2013) 251801 [arXiv:1304.6335] [INSPIRE].

[16] MINOS collaboration, Electron neutrino and antineutrino appearance in the full MINOS data sample, Phys. Rev. Lett. 110 (2013) 171801 [arXiv:1301.4581] [INSPIRE].

[17] T2K collaboration, Measurement of neutrino and antineutrino oscillations by the T2K experiment including a new additional sample of $\nu_{e}$ interactions at the far detector, Phys. Rev. D 96 (2017) 092006 [Erratum ibid. D 98 (2018) 019902] [arXiv:1707.01048] [INSPIRE].

[18] T2K collaboration, Search for CP-violation in Neutrino and Antineutrino Oscillations by the T2K Experiment with $2.2 \times 10^{21}$ Protons on Target, Phys. Rev. Lett. 121 (2018) 171802 [arXiv: 1807.07891] [INSPIRE].

[19] NOvA collaboration, Constraints on Oscillation Parameters from $\nu_{e}$ Appearance and $\nu_{\mu}$ Disappearance in NOvA, Phys. Rev. Lett. 118 (2017) 231801 [arXiv:1703.03328] [INSPIRE].

[20] NOvA collaboration, New constraints on oscillation parameters from $\nu_{e}$ appearance and $\nu_{\mu}$ disappearance in the NOvA experiment, Phys. Rev. D 98 (2018) 032012 [arXiv:1806.00096] [INSPIRE]. 
[21] DaYa BAY collaboration, Measurement of electron antineutrino oscillation with 1958 days of operation at Daya Bay, Phys. Rev. Lett. 121 (2018) 241805 [arXiv: 1809.02261] [INSPIRE].

[22] RENO collaboration, Measurement of Reactor Antineutrino Oscillation Amplitude and Frequency at RENO, Phys. Rev. Lett. 121 (2018) 201801 [arXiv:1806.00248] [INSPIRE].

[23] Double CHOOZ collaboration, Improved measurements of the neutrino mixing angle $\theta_{13}$ with the Double CHOOZ detector, JHEP 10 (2014) 086 [Erratum ibid. 02 (2015) 074] [arXiv: 1406.7763] [INSPIRE].

[24] M. Dentler, Á. Hernández-Cabezudo, J. Kopp, M. Maltoni and T. Schwetz, Sterile neutrinos or flux uncertainties? - Status of the reactor anti-neutrino anomaly, JHEP 11 (2017) 099 [arXiv: 1709. 04294] [INSPIRE].

[25] Daya Bay collaboration, Improved Measurement of the Reactor Antineutrino Flux and Spectrum at Daya Bay, Chin. Phys. C 41 (2017) 013002 [arXiv: 1607.05378] [INSPIRE].

[26] Borexino collaboration, Comprehensive measurement of pp-chain solar neutrinos, Nature 562 (2018) 505 [INSPIRE].

[27] ICECuBE collaboration, Determining neutrino oscillation parameters from atmospheric muon neutrino disappearance with three years of IceCube DeepCore data, Phys. Rev. D 91 (2015) 072004 [arXiv: 1410.7227] [INSPIRE].

[28] ICECuBE collaboration, IceCube Oscillations: 3 years muon neutrino disappearance data, http://icecube.wisc.edu/science/data/nu_osc.

[29] Super-Kamiokande collaboration, Atmospheric neutrino oscillation analysis with external constraints in Super-Kamiokande I-IV, Phys. Rev. D 97 (2018) 072001 [arXiv:1710.09126] [INSPIRE].

[30] SuperKamiokande collaboration, Atmospheric neutrino oscillation analysis with external constraints in Super-Kamiokande I-IV, link to data release:

http://www-sk.icrr.u-tokyo.ac.jp/sk/publications/result-e.html\#atmosci2018 (2018).

[31] IceCube collaboration, Measurement of Atmospheric Neutrino Oscillations at 6-56 GeV with IceCube DeepCore, Phys. Rev. Lett. 120 (2018) 071801 [arXiv:1707.07081] [InSPIRE].

[32] J. Elevant and T. Schwetz, On the determination of the leptonic CP phase, JHEP 09 (2015) 016 [arXiv: 1506.07685] [INSPIRE].

[33] M.C. Gonzalez-Garcia and C. Pena-Garay, Three neutrino mixing after the first results from K2K and KamLAND, Phys. Rev. D 68 (2003) 093003 [hep-ph/0306001] [INSPIRE].

[34] P.I. Krastev and S.T. Petcov, Resonance Amplification and $t$ Violation Effects in Three Neutrino Oscillations in the Earth, Phys. Lett. B 205 (1988) 84 [INSPIRE].

[35] C. Jarlskog, Commutator of the Quark Mass Matrices in the Standard Electroweak Model and a Measure of Maximal CP-violation, Phys. Rev. Lett. 55 (1985) 1039 [INSPIRE].

[36] Particle Data Group collaboration, Review of Particle Physics, Phys. Rev. D 98 (2018) 030001 [INSPIRE].

[37] J. Bergstrom, M.C. Gonzalez-Garcia, M. Maltoni, C. Pena-Garay, A.M. Serenelli and N. Song, Updated determination of the solar neutrino fluxes from solar neutrino data, JHEP 03 (2016) 132 [arXiv: 1601.00972] [INSPIRE].

[38] N. Vinyoles et al., A new Generation of Standard Solar Models, Astrophys. J. 835 (2017) 202 [arXiv: 1611.09867] [INSPIRE]. 
[39] L. Wolfenstein, Neutrino Oscillations in Matter, Phys. Rev. D 17 (1978) 2369 [InSPIRE].

[40] S.P. Mikheyev and A. Yu. Smirnov, Resonance Amplification of Oscillations in Matter and Spectroscopy of Solar Neutrinos, Sov. J. Nucl. Phys. 42 (1985) 913 [inSPIRE].

[41] M. Ikeda, Solar neutrino measurements with Super-Kamiokande, talk given at the XXVIII International Conference on Neutrino Physics and Astrophysics, Heidelberg, Germany, June 4-9, 2018.

[42] Y. Nakano, ${ }^{8}$ B solar neutrino spectrum measurement using Super-Kamiokande IV, Ph.D. Thesis, Tokyo U. (2016).

[43] N. Okamura, Effect of the smaller mass-squared difference for the long base-line neutrino experiments, Prog. Theor. Phys. 114 (2006) 1045 [hep-ph/0411388] [INSPIRE].

[44] H. Nunokawa, S.J. Parke and R. Zukanovich Funchal, Another possible way to determine the neutrino mass hierarchy, Phys. Rev. D 72 (2005) 013009 [hep-ph/0503283] [INSPIRE].

[45] M. Wascko, T2k status, results, and plans, June 2018 [DOI:10.5281/zenodo.1286752].

[46] M. Sanchez, Nova results and prospects, June 2018 [DOI:10.5281/zenodo.1286758].

[47] V.D. Barger, K. Whisnant, S. Pakvasa and R.J.N. Phillips, Matter Effects on Three-Neutrino Oscillations, Phys. Rev. D 22 (1980) 2718 [InSPIRE].

[48] H. Minakata, H. Nunokawa, S.J. Parke and R. Zukanovich Funchal, Determining neutrino mass hierarchy by precision measurements in electron and muon neutrino disappearance experiments, Phys. Rev. D 74 (2006) 053008 [hep-ph/0607284] [INSPIRE].

[49] M. Blennow and T. Schwetz, Determination of the neutrino mass ordering by combining PINGU and Daya Bay II, JHEP 09 (2013) 089 [arXiv: 1306.3988] [INSPIRE].

[50] ICECUBE collaboration, Measurement of atmospheric neutrino oscillations with three years of data from the full sky, https://icecube.wisc.edu/science/data/2018nuosc (2018).

[51] F. Halzen, Conclusion: how the past history can shed light on the future of neutrinos, talk given at the History of the Neutrino Conference, Paris, France, September 5-7, 2018.

[52] J. Bonn et al., The Mainz neutrino mass experiment, Nucl. Phys. Proc. Suppl. 91 (2001) 273 [INSPIRE].

[53] KATRIN collaboration, KATRIN: A Next generation tritium beta decay experiment with sub-eV sensitivity for the electron neutrino mass. Letter of intent, hep-ex/0109033 [INSPIRE].

[54] GERDA collaboration, Improved Limit on Neutrinoless Double- $\beta$ Decay of ${ }^{76}$ Ge from GERDA Phase II, Phys. Rev. Lett. 120 (2018) 132503 [arXiv:1803.11100] [InSPIRE].

[55] KamLAND-Zen collaboration, Search for Majorana Neutrinos near the Inverted Mass Hierarchy Region with KamLAND-Zen, Phys. Rev. Lett. 117 (2016) 082503 [arXiv: 1605.02889] [INSPIRE].

[56] J.J. Gomez-Cadenas, J. Martin-Albo, M. Mezzetto, F. Monrabal and M. Sorel, The Search for neutrinoless double beta decay, Riv. Nuovo Cim. 35 (2012) 29 [arXiv:1109.5515] [INSPIRE].

[57] G.L. Fogli et al., Observables sensitive to absolute neutrino masses: Constraints and correlations from world neutrino data, Phys. Rev. D 70 (2004) 113003 [hep-ph/0408045] [INSPIRE]. 
[58] S. Pascoli, S.T. Petcov and T. Schwetz, The Absolute neutrino mass scale, neutrino mass spectrum, Majorana CP-violation and neutrinoless double-beta decay, Nucl. Phys. B 734 (2006) 24 [hep-ph/0505226] [INSPIRE].

[59] B.T. Cleveland et al., Measurement of the solar electron neutrino flux with the Homestake chlorine detector, Astrophys. J. 496 (1998) 505 [INSPIRE].

[60] F. Kaether, W. Hampel, G. Heusser, J. Kiko and T. Kirsten, Reanalysis of the GALLEX solar neutrino flux and source experiments, Phys. Lett. B 685 (2010) 47 [arXiv:1001.2731] [INSPIRE].

[61] SAGE collaboration, Measurement of the solar neutrino capture rate with gallium metal. III: Results for the 2002-2007 data-taking period, Phys. Rev. C 80 (2009) 015807 [arXiv: 0901.2200] [INSPIRE].

[62] Super-Kamiokande collaboration, Solar neutrino measurements in Super-Kamiokande-I, Phys. Rev. D 73 (2006) 112001 [hep-ex/0508053] [INSPIRE].

[63] Super-Kamiokande collaboration, Solar neutrino measurements in Super-Kamiokande-II, Phys. Rev. D 78 (2008) 032002 [arXiv:0803.4312] [INSPIRE].

[64] Super-Kamiokande collaboration, Solar neutrino results in Super-Kamiokande-III, Phys. Rev. D 83 (2011) 052010 [arXiv: 1010.0118] [InSPIRE].

[65] SNO collaboration, Combined Analysis of all Three Phases of Solar Neutrino Data from the Sudbury Neutrino Observatory, Phys. Rev. C 88 (2013) 025501 [arXiv:1109.0763] [INSPIRE].

[66] G. Bellini et al., Precision measurement of the 7Be solar neutrino interaction rate in Borexino, Phys. Rev. Lett. 107 (2011) 141302 [arXiv:1104.1816] [INSPIRE].

[67] Borexino collaboration, Measurement of the solar $8 B$ neutrino rate with a liquid scintillator target and $3 \mathrm{MeV}$ energy threshold in the Borexino detector, Phys. Rev. D 82 (2010) 033006 [arXiv: 0808.2868] [INSPIRE].

[68] BorEXINo collaboration, Neutrinos from the primary proton-proton fusion process in the Sun, Nature 512 (2014) 383 [INSPIRE].

[69] M. Honda, M. Sajjad Athar, T. Kajita, K. Kasahara and S. Midorikawa, Atmospheric neutrino flux calculation using the NRLMSISE-00 atmospheric model, Phys. Rev. D 92 (2015) 023004 [arXiv: 1502.03916] [INSPIRE].

[70] KamLAND collaboration, Reactor On-Off Antineutrino Measurement with KamLAND, Phys. Rev. D 88 (2013) 033001 [arXiv: 1303.4667] [INSPIRE].

[71] A. Cabrera Serra, Double Chooz Improved Multi-Detector Measurements, talk given at the CERN EP colloquium, CERN, Switzerland, September 20, 2016.

[72] A. Izmaylov, T2K Neutrino Experiment. Recent Results and Plans, talk given at the Flavour Physics Conference, Quy Nhon, Vietnam, August 13-19, 2017.

[73] T. Koga, Measurement of neutrino interactions on water and search for electron anti-neutrino appearance in the T2K experiment, Ph.D. Thesis, Tokyo U. (2018) [https://www.t2k.org/docs/thesis/090/Doctor\%20thesis].

[74] S. Dennis, Muon Antineutrino Disappearance and Non-Standard Interactions at the T2K Experiment, Ph.D. Thesis, Warwick U. (2015). 
[75] M. BAIRD, Reconstructing Neutrino Energies with the NOvA Detectors, June, 2018 [DOI:10.5281/zenodo.1301120].

[76] A. Himmel, First Oscillation Results with Neutrino and Antineutrino Beams in NOvA, talk given at the Joint Theoretical-Experimental Physics Seminar, Fermilab, U.S.A., June 15, 2018.

[77] DAYA BAY collaboration, Measurement of electron antineutrino oscillation based on 1230 days of operation of the Daya Bay experiment, Phys. Rev. D 95 (2017) 072006 [arXiv: 1610.04802] [INSPIRE].

[78] P. Vogel and J.F. Beacom, Angular distribution of neutron inverse beta decay, $\bar{\nu}_{e}+p \rightarrow e^{+}+n$, Phys. Rev. D 60 (1999) 053003 [hep-ph/9903554] [INSPIRE].

[79] P. Huber, On the determination of anti-neutrino spectra from nuclear reactors, Phys. Rev. C 84 (2011) 024617 [Erratum ibid. C 85 (2012) 029901] [arXiv: 1106. 0687] [INSPIRE].

[80] T.A. Mueller et al., Improved Predictions of Reactor Antineutrino Spectra, Phys. Rev. C 83 (2011) 054615 [arXiv: 1101.2663] [INSPIRE].

[81] RENO collaboration, Fuel-composition dependent reactor antineutrino yield and spectrum at RENO, arXiv:1806.00574 [INSPIRE].

[82] RENO collaboration, RENO: An Experiment for Neutrino Oscillation Parameter $\theta_{13}$ Using Reactor Neutrinos at Yonggwang, arXiv:1003.1391 [INSPIRE]. 